\title{
Proximal Tissues and Patterned Neurite Outgrowth at the Lumbosacral Level of the Chick Embryo: Deletion of the Dermamyotome
}

\author{
KATHRYN W. TOSNEY \\ Department of Biology, University of Michigan, Ann Arbor, Michigan 48109 \\ Received January 7, 1987; accepted in revised form March 30, 1987
}

\begin{abstract}
The role of the dermamyotome (the dorsal portion of the somite which gives rise to muscles and dermis) in the development of patterned axon outgrowth was examined under conditions where limb development was substantially undisturbed. One or more chick dermamyotomes were removed before or during early neurite outgrowth and subsequent development was examined. Several developmental processes suspected to depend on the dermamyotome were not altered by its removal: (1) Neural crest cells that form sensory ganglia migrated and condensed in their normal segmental pattern. (2) The distal progression, dorsal-ventral organization, and segmentation of spinal nerves were unaltered. (3) Motoneuron pathway selection and projection patterns in the limb were normal in all respects.

The most interesting finding was that the formation of the dorsal ramus is dependent on the nearby dermamyotome which provides the targets for this nerve. When a single or two adjacent dermamyotomes were removed, the metameric epaxial muscles derived from each dermamyotome were absent and the dorsal ramus extended into epaxial muscle in the closest adjacent segment. However, when dermamyotomes in both adjacent segments had also been removed or substantially reduced, the dorsal ramus did not form. These results strongly suggest that the target provides a chemotactic signal for proper outgrowth of dorsal ramus axons. (c) 1987 Academic Press, Inc.
\end{abstract}

\section{INTRODUCTION}

Neurite outgrowth in the lumbosacral region of the developing chick embryo is patterned in two senses: both a stereotyped gross anatomical nerve pattern and a specific projection pattern emerge during development. The gross anatomical nerve pattern may be stereotyped because it depends on the stereotyped development of the surrounding embryonic tissues. Growth cones, the growing tips of neurites, may find some tissues more permissive for outgrowth and use these as general pathways (Lance-Jones and Landmesser, 1980; Summerbell and Stirling, 1981; Lewis et al., 1983; Tosney and Landmesser, 1984, 1985a). Indeed, the local embryonic environment is neccessary and sufficient for development of a normal gross anatomical nerve pattern (Tosney and Landmesser, 1984), suggesting that the pathways are delineated by elements within reach of growth cone filipodia. When the general pathways branch, a specific projection pattern emerges as growth cones select the proper paths (see Tosney and Landmesser, 1985b). Because they can select correct paths following many experimental alterations in the environment, specific cues must be postulated to guide their pathway choices (Lance-Jones and Landmesser, 1980, 1981a; Ferguson, 1983; Whitelaw and Hollyday, 1983a; Tosney and Landmesser, 1984; Lance-Jones, 1986, 1987; see Landmesser,
1985,1986 for reviews). It is important to determine the sources and modes of action of both the permissive and the specific navigational cues. What cues are provided by tissues along or adjacent to the pathways? Are some cues target-related and chemotactic in nature?

The somites are the most prominant and experimentally accessible structures in the proximal environment. Shortly before axon outgrowth, each somite undergoes morphogenesis to form a dermamyotome (the dorsal portion of a somite, which will give rise to skeletal muscles and dermis) and sclerotome (the ventral portion of a somite, which will give rise to vertebral cartilage). The relative contributions of each of these tissues to permissive and specific types of guidance is unclear. The strategy I have taken to examine this issue is to selectively remove the dermamyotome and examine the gross anatomy and specificity of the innervation that develops in its absence.

One striking aspect of the gross anatomical nerve pattern is the segmentation of spinal nerves and ganglia. The somites have long been regarded as important to the development of this segmentation (Detwiler, 1934). While the anterior portion of each somite has been shown to be preferred for axonal outgrowth (Keynes and Stern, 1984), the relative contributions of somite derivatives to segmentation are unclear and have been ascribed to both the dermamyotome (Detwiler, 1934; Lor- 
ing and Erickson, 1986) and the sclerotome (Keynes and Stern, 1984; Rickman et al., 1985; Tosney and Landmesser, 1985a; Stern et al., 1986). This controversy can be at least partially resolved by determining whether dorsal root ganglia (DRG) and spinal nerves form in a segmental pattern in the absence of the dermamyotome.

A second, relatively unexamined aspect of the gross patterning of spinal nerves is their organization along the dorsal-ventral axis. The spinal nerve normally grows out as a coherent sheet through the anterior sclerotome (Tosney and Landmesser, 1985a). The restriction of outgrowth along the dorsal-ventral plane has been ascribed to the growth cones' use of the planar undersurface of the dermamyotome as a substratum (Bennett et al., 1980; Bennett, 1983; see also Hollyday, 1983), to their preference for neurites as a substratum (Tosney and Landmesser, 1985a; Tosney et al., 1986), and to their avoidance of the glycosaminoglycan-rich ventral sclerotome (Tosney and Landmesser, 1985a). I addressed this issue by examining the dorsal-ventral coherence of spinal nerves following dermamyotome deletion.

The ordered distal outgrowth of spinal nerves, in which axons grow directly to the limb base with few alterations in their trajectory, is another aspect of patterning that is little understood. Chemotaxis to distal tissues is not required for distal outgrowth because axons enter the limb when limb muscles are substantially reduced (Lewis et al., 1981, Tosney, 1986) or absent (Phelan and Hollyday, 1986) and, in fact, spinal nerves form normally when the entire limb is absent (Tosney and Landmesser, 1984). While the anterior portion of the sclerotome appears to be the environment directly within reach of growth cone filipodia (Tosney and Landmesser, 1985a), it is also possible that the undersurface of the dermamyotome orients distal outgrowth (see Bennett et al., 1980; Bennett, 1983; Hollyday, 1983). Other possibilities include the strong tendency of axons to fasciculate, coupled with forward persistence of growth cone movement (Tosney and Landmesser, 1985a; Tosney et al., 1986) or guidance by neural crest cells (Carpenter and Hollyday, 1986; Tanaka and Landmesser, 1987; Landmesser, 1987). To address this issue, I determined whether the distal outgrowth of spinal nerves was altered by dermamyotome deletion.

Proximal tissues may also provide specific cues for axonal pathway choice, for instance, when axons enter dorsal or ventral nerve trunks as they emerge from the nerve plexus at the limb base. Proximal cues appear to be sufficient for this pathway decision, since axons can make the decision properly even in the absence of all tissues distal to the limb girdle (Tosney and Landmesser, 1984) or when the target complement in the limb has been altered (Lance-Jones, 1986; Whitelaw and Hollyday, 1983a,b). In addition, axons sort out into the correct paths even when they have been displaced along the anterior-posterior axis (Lance-Jones and Landmesser, 1980 , 1981a), suggesting that the essential cues are equivalent at all axial levels. The dermamyotome is segmentally repeated and is close enough to the plexus region to be an effective source of a chemical cue (Tosney and Landmesser, 1985a; see Crick, 1970). If the dermamyotome were the source of essential positional information at the limb base, then axonal pathway selection and specific projection within the limb should be altered in its absence.

I was also able to address specific target-related cues by examining the development of a branch of the spinal nerve, the dorsal ramus, which innervates epaxial muscles (the intervertebral and back muscles, see Tosney, 1987a). The axons in this nerve quickly diverge from the spinal nerve and travel a short distance to their epaxial muscle target. Although both epaxial and limb muscles arise from the dermamyotome, they do so by different modes. During normal development, the epaxial muscles (as well as the intercostal muscles) arise in situ from each dermamyotome: in each segment, epaxial muscle is made up entirely of cells that arose within that segment (Chevallier, 1979). In contrast, limb muscles arise from precursor cells in the dermamyotome that migrate distally and form premuscle masses within the limb; individual muscles cleave from the muscle masses after they have been innervated (Chevallier et al., 1978; Chevallier, 1979; Beresford, 1983; Christ et al., 1983; LanceJones, 1987). Limb muscles are seldom affected by removal of a few dermamyotomes because a significant proportion of their precursors migrate before the dermamyotome becomes histologically distinct (Chevallier, 1978; Jacob et al., 1979). In addition, each hindlimb muscle generally receives contributions from 2-5 dermamyotomes (Lance-Jones, 1987). Even when a significant fraction of the precursor population has been removed, the remaining cells can regulate to form a normal complement of limb muscles (Chevallier et al., 1977; see also Mauger and Kieny, 1980). Given the extensive ability of limb muscle precursors to compensate for removed tissue, it was somewhat surprising to find that epaxial muscle was absent in a segment when I had completely deleted a single dermamyotome. Neither dermamyotome nor maturing epaxial muscles compensated for the missing tissue by spreading into the deprived segment. The differential ability of limb and epaxial muscle precursors to regulate is probably related to their different modes of origin: migrating precursors give rise to limb but not epaxial muscles. Although it is not known why epaxial muscle precursors fail to regulate across segment boundaries, it was convenient for this study. Since dermamyotome deletion had different consequences for the development of epaxial and limb muscles, I was able to 
examine the development of the dorsal ramus in the absence of its target without significantly affecting any possible target dependence of axons that enter the limb.

\section{MATERIALS AND METHODS}

Embryonic surgeries. White Leghorn eggs of 2-4 days incubation were candled, $2 \mathrm{ml}$ of albumin was removed with a sterile syringe and needle, and the eggs were returned to the incubator for $2-4 \mathrm{hr}$ to allow the embryos to settle. Shell $\left(1 \mathrm{~cm}^{2}\right)$ was removed from above the embryo with a dental drill, 2 drops of Tyrodes solution containing 100 units $/ \mathrm{ml}$ of penicillin-streptomycin (GIBCO) was added, the window was sealed with surgical tape and the embryos were incubated for 1-4 hr. Embryos were then stained with a drop of $0.2 \%$ neutral red in distilled water and staged using Hamburger and Hamilton (1951) criteria, and the vitelline membrane was opened. Because the dermamyotome is a more densely packed tissue than the underlying sclerotome, it stained more darkly with neutral red and could be casily distinguished from underlying sclerotome which was identifiable as a more lightly stained coherent sphere. A small cut was made in the ectoderm lateral or posterior to the operation site using a fine tungsten needle and one or more dermamyotomes were removed with a small micropipet (tip size 20-50 $\mu \mathrm{m}$ ) attached to polyethylene tubing. Deletions were performed from thoracic level 5 (T5) through lumbosacral level 6 (LS6), corresponding approximately to somites 22 through 31 . Eggs were sealed with surgical tape and incubated until the desired stage.

Operations were performed on 87 embryos; 71 survived the operation and 9 of these were discarded due to abnormalities associated with faulty amnion closure or dehydration during the operation. The embryos analyzed included 35 with single and 27 with 2 or more adjacent dermamyotomes removed. An additional series of approximately 50 embryos done when I was gaining proficiency with the operation are not included in the present analysis, although their development is consistent with the results reported. The initial series established that the dermamyotome could be excised fairly cleanly from somites that were two or more somites anterior to the unsegmented somitic plate. Farlier excision consistently resulted in regulation or in concurrent reduction of sclerotomal derivatives as well. A subsequent paper in this series examines the effects of removing part or all of the sclerotome as well as the dermamyotome (Tosney, 1987b).

In order to determine whether the dermamyotome was essential during some critical period, operations were performed during three stage groups: early stage 17 when dermamyotomes first appear and before axon out- growth begins $(N=36)$, stage $17^{+}$to 18 during the earliest period of axon outgrowth $(N=15)$, and stages 20 21 as the first axons reach the plexus region $(N=11)$. In addition, embryos were also classified by the phase of crest migration (using the criteria of Loring and $\mathrm{Er}^{-}$ ickson, 1987, and Rickman et al., 1985) by determining the distance between the operated segment and the segmental plate. Thus, operations were performed 1-6 somites anterior to the unsegmented plate when neural crest has not yet invaded the somite ( $N=77$ segments), 7-9 somites anterior to the unsegmented plate as the first crest cells move beneath the dermamyotome $(N$ $=15$ segments), and at more mature levels after crest had begun to accumulate within the sclerotome $(N=13$ segments). In fact, distinct stage-dependent differences were not found and for most purposes the results were pooled.

Analysis of neurite projection patterns. The ventral roots, spinal nerves, nerve trunks, or muscles of 47 of the operated embryos were injected with horseradish pcroxidase (HRP) and processed by previously published procedures (Landmesser, 1978; Tosney and Landmesser, 1984) to visualize individual axons during outgrowth and to assess the specificity of axonal projection. The dorsal ramus is a tiny nerve that is difficult to inject in normal embryos, and I did not try to inject it in operated embryos; outgrowth of motoneurons into this ramus was assessed by ventral root injection. In addition, 15 operated embryos were fixed without laminectomy in Bouin's, postfixed in Carnoy's overnight, paraffin embedded, sectioned at $12 \mu \mathrm{m}$ perpendicular to the spinal cord, and stained in alcian blue and eosin or in cresyl violet. Examination of these specimens eliminated the possibility that axons took unusual routes through tissue that is normally removed during processing.

Embryos were fixed for examination in four groups for assessment of different aspects of patterned outgrowth: (1) within 1-6 hr of the operation $(N=4)$, to verify the continued accuracy of deletion; (2) during the period in which growth cones traverse the proximal environment and gather at the leg base (stages 21-25, $N$ $=30$ ), to determine whether dorsal ramus axons leave the spinal nerve in the absence of nearby target and whether axons ramify more extensively during spinal nerve formation; (3) at a stage when motoneuron axons have sorted out along the dorsal-ventral axis at the leg base and muscle nerves have begun to form (stage 26 , $N=20$ ), to examine sorting out in the plexus region, the precision of dorsal or ventral nerve trunk pathway choice, and the initial precision of innervation; and (4) following establishment of "mature" limb innervation (stages 29-34, $N=8$ ), to examine the precision of innervation of limb muscles. Before removing ectoderm at the latter stages (to facilitate oxygen diffusion during 
incubation for HRP transport) the distribution of feather germs was determined. These were generally absent dorsal to the operation site, showing that dermis (which induces feather germs) had been effectively removed.

Analysis of the anatomy of operated embryos. The dermamyotome in normal embryos undergoes morphogenesis to form the myotome (which gives rise to all skeletal muscle at thoracic levels and to epaxial musculature at lumbosacral levels; Chevallier, 1979) and the dermatome (which gives rise to the axial dermis (Mauger, 1972) and the precursor cells for limb and abdominal muscles; Chevallier el al., 1978; Chevallier, 1979; Beresford, 1983; Christ et al., 1983; Lance-Jones, 1987). Since the dermis could be distinguished from other mesenchyme only by position, the presence of the myotome or the muscles that were derived from it in situ was used to determine the degree of deletion in each operated embryo.

Suitably fine criteria for detecting tiny fragments of myotome and small nerve branches were provided by the discovery that when embryos are processed as above (with or without $\mathrm{DAB}$ treatment) and viewed with fluorescein epifluorescent optics (Nikon filter set B, excitation $450-490 \mathrm{~nm}$, barrier filter $520 \mathrm{~nm}$ ) cells of the myotomes and muscles as well as neurons and axons fluoresce bright yellow and are clearly visible against a background of brownish cells. Myotomal remnants of as few as a dozen cells could be reliably visualized and 31 of the embryos displayed complete removal of one or more dermamyotomes by this criterion. Red blood cells could be distinguished from isolated myotomal cells by their cell shape and inclusion in vascular endothelia. The mesonephros and some cells around condensed cartilage in older embryos also fluoresced. It is not known what causes this convenient autofluorescence.

Complete reconstructions of the gross anatomical nerve pattern and the distribution of dorsal root ganglia (DRG), epaxial muscle (or myotome), and labeled somata and axons were made using a camera lucida. In $11 \mathrm{em}-$ bryos (stage 26 or older) in which HRP had been injected into individual spinal cord segments, ventral roots, or spinal nerves, the segmental specificity of projection was determined by observation of individual muscle nerves at approximately $600 \times$ magnification. Seventeen nonoverlapping pools for individual muscles or muscle groups were mapped in 14 additional embryos. The relative size of DRG on operated and contralateral sides was assessed in all operated embryos. To determine whether contralateral DRG were reduced in size, each was compared to DRG of comparable thoracic or lumbosacral segments outside the operated region; experimental DRG were compared to the same standard. Since many neurons have yet to differentiate during the stages studied and reliable cell counts cannot be obtained, only obvious reductions in ganglion volume were noted. Size was determined by comparing the extent of each DRG on anterior-posterior, dorsal-ventral, and medial-lateral axes and DRG were classified as normal, small (reduced in size by $\frac{1}{4}$ or more), or absent.

\section{RESULTS}

Development of nonmuscle tissues in operated embryos. A dermamyotome could be cleanly removed shortly after it had separated from the sclerotome (Fig. 1A) or at later stages when it had begun to form dermatome and myotome (Fig. 1B). Tissues adjacent to the deleted dermamyotome were usually minimally disturbed and in many cases even the neural crest and ectoderm remained substantially intact.

Several morphological features show that the dermatome and dermis did not develop in the operated segment following dermamyotome deletion. One of these is the absence of feather germs above the operation site which was observed before removing the ectoderm from older embryos. In younger embryos, ectoderm was not removed during processing and it was obvious that the subectodermal mesenchyme had been replaced by a 20to $30-\mu \mathrm{m}$ space filled with alcian-blue positive material (Figs. 2A and 2B). The space was distinct in 33 segments where the myotome was completely removed and was not detectable in 5 additional segments. When a fragment of myotome remained the space was dorsal to the region of successful myotome removal ( $N=23$ segments) or was not distinct ( $N=12$ segments). In no case did the space extend beneath the ectoderm at limb or lateral trunk regions (Fig. $2 \Lambda$ ), confirming descriptive (Christ et al., 1983) and experimental (Mauger, 1972) evidence that the dermamyotome does not contribute dermis to the lateral epithelium.

When a dermamyotome was completely removed, the morphogenesis of the sclerotome to form vertebrae was in most cases (50/62 segments) surprisingly normal in embryos examined up to stage 28. In some older embryos, the vertebrae were substantially normal (Fig. 3A) while in others the dorsal vertebral cartilage had condensed in an abnormal pattern and spaces were occassionally seen adjacent to the spinal cord or between the vertebrae and pelvic girdle (Fig. 3B). These anomalies may have been associated with inadvertent removal of dorsal sclerotome. Since lateral and ventral vertebrae were generally normal, the morphogenesis of the sclerotome may be largely independent of the presence of the dermamyotome by stage 17 , barring direct damage to the tissue.

Muscle development in operated embryos. Limb muscles were never obviously depleted even at early stages. For instance, in Fig. 2C, the dorsal muscle masses on exper- 

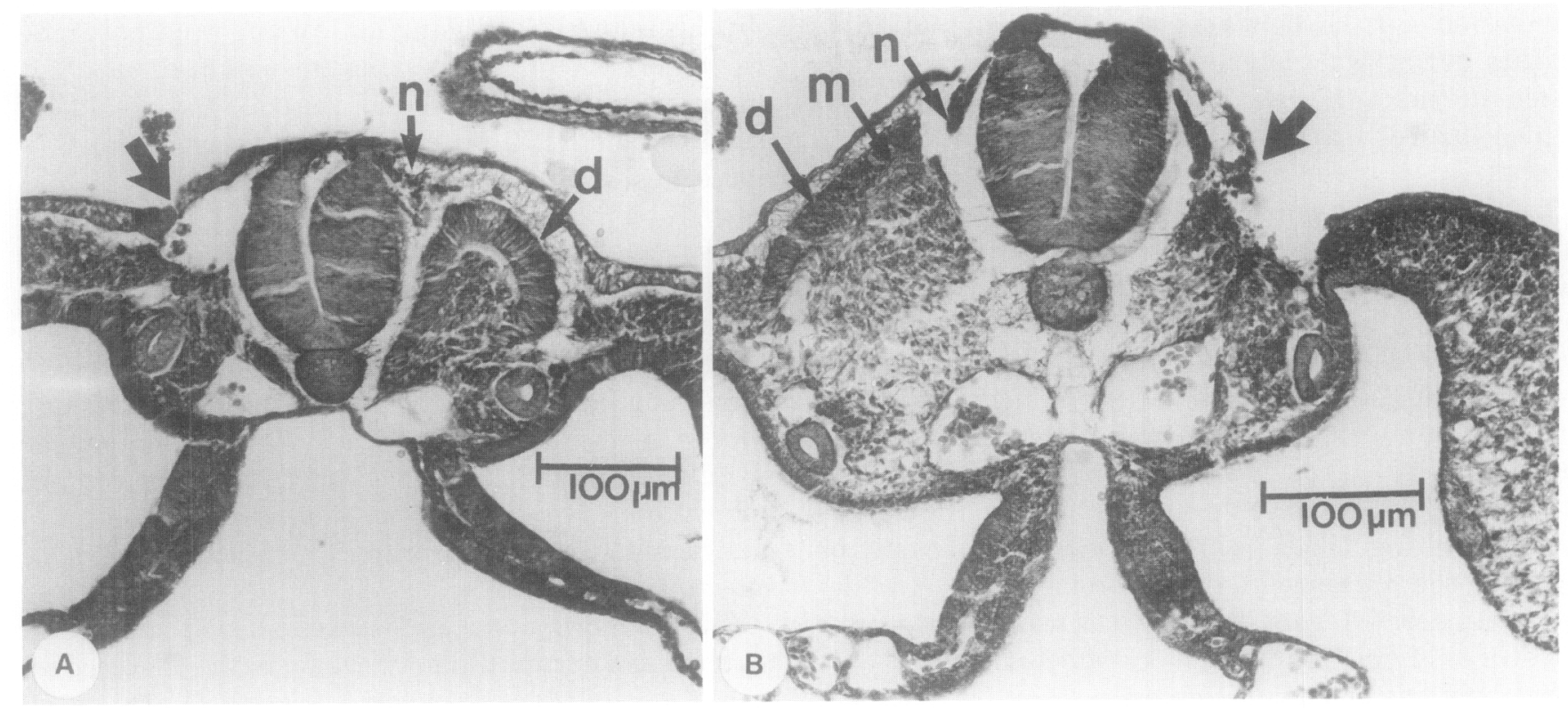

FIG. 1. Early and late dermamyotome deletions. (A) On the control side (right) the dermamyotome (d) is in the process of separation from the sclerotome and the earliest neural crest cells ( $n$ ) have begun to migrate into a cell-free space filled with extracellular matrix, stained here with alcian blue. On the left, the dermamyotome was removed through a lateral ectodermal slit (arrow) which began to heal $4 \mathrm{hr}$ after the operation. The sclerotome appears to be intact, but the earliest migrating neural crest cells have been removed in this embryo. (B) On the control side (left) the dermamyotome is beginning to form a distinct dermatome (d) and myotome (m) and neural crest is migrating as a compact stream ( $n$ ). The neural crest has been preserved on the experimental side. This is one of the few embryos in which the ectoderm was removed as well as the dermamyotome (large arrow); however, the ectoderm began to regenerate $1 \mathrm{hr}$ after the operation. Light micrographs, alcian blue and eosin.

imental and control sides are similar in size and position at stage 26 following deletion of 3 dermamyotomes at stage 17. The full complement of limb muscles always developed at later stages. These were not noticeably reduced in size even when dermamyotomes were removed that bracketed the crural region from which the anterior thigh muscles are derived (segments T7 or LS1 through LS3; $N=4$ ) or the central region of the leg (LS2 to LS4 or LS6; $N=2$ ). For instance, in Fig. 3B the small muscles immediately distal to the pelvic girdle are normal. These observations are not surprising, since some precursors for limb muscles had migrated from the dermamyotome before the operation (see Chevallier, 1978; Jacob et al., 1979 ) and these would have regulated to give rise to normal limb muscles (see Chevallier et al., 1977).

At lumbosacral levels complete removal of a single dermamyotome resulted in the absence of the myotome (Fig. 2C) or, in older embryos, epaxial muscles (the segmental muscles of the back and vertebrae; Figure 3B) in that segment. The dermamyotome in adjoining segments did not compensate by extending into the deleted area. This is consistent with the fact that the dermamyotome normally gives rise to all of its derivatives (except limb muscle) in situ (Chevallier, 1979). However, during normal development the individual segments of the dorsal epaxial muscle normally fuse at segment bor- ders to form the long muscle of the back (Tosney, 1987a). It was thus surprising to find that maturing epaxial muscle did not extend into the deprived segment at later stages. Instead, at the border of each deletion, the epaxial muscle fibers were invariably oriented mediolaterally as though they were turning about on themselves (see Fig. 11C). It is possible that the dermis plays an important role in epaxial muscle development and that muscle could not colonize adjacent to deprived segments when dermis was absent.

When the dermamyotome was incompletely removed, the fragments often developed in a position-dependent pattern. The dorsomedial dermamyotome normally contributes to the back (longissimus dorsi) muscle while more ventrolateral dermamyotome contributes to intervertebral muscle (Tosney, 1987a). In several cases only one of these muscles was represented and the few myofibrils present were organized appropriately for that muscle. This suggests that each epaxial muscle develops from a local region of the dermamyotome and forms independently of other muscles.

The intercostal muscles also arise metamerically, from individual dermamyotomes (Chevallier, 1979). Like the epaxial muscles, these did not develop in the deprived segment when the dermamyotome had been completely removed $(N=6)$. However, it was often difficult to totally 

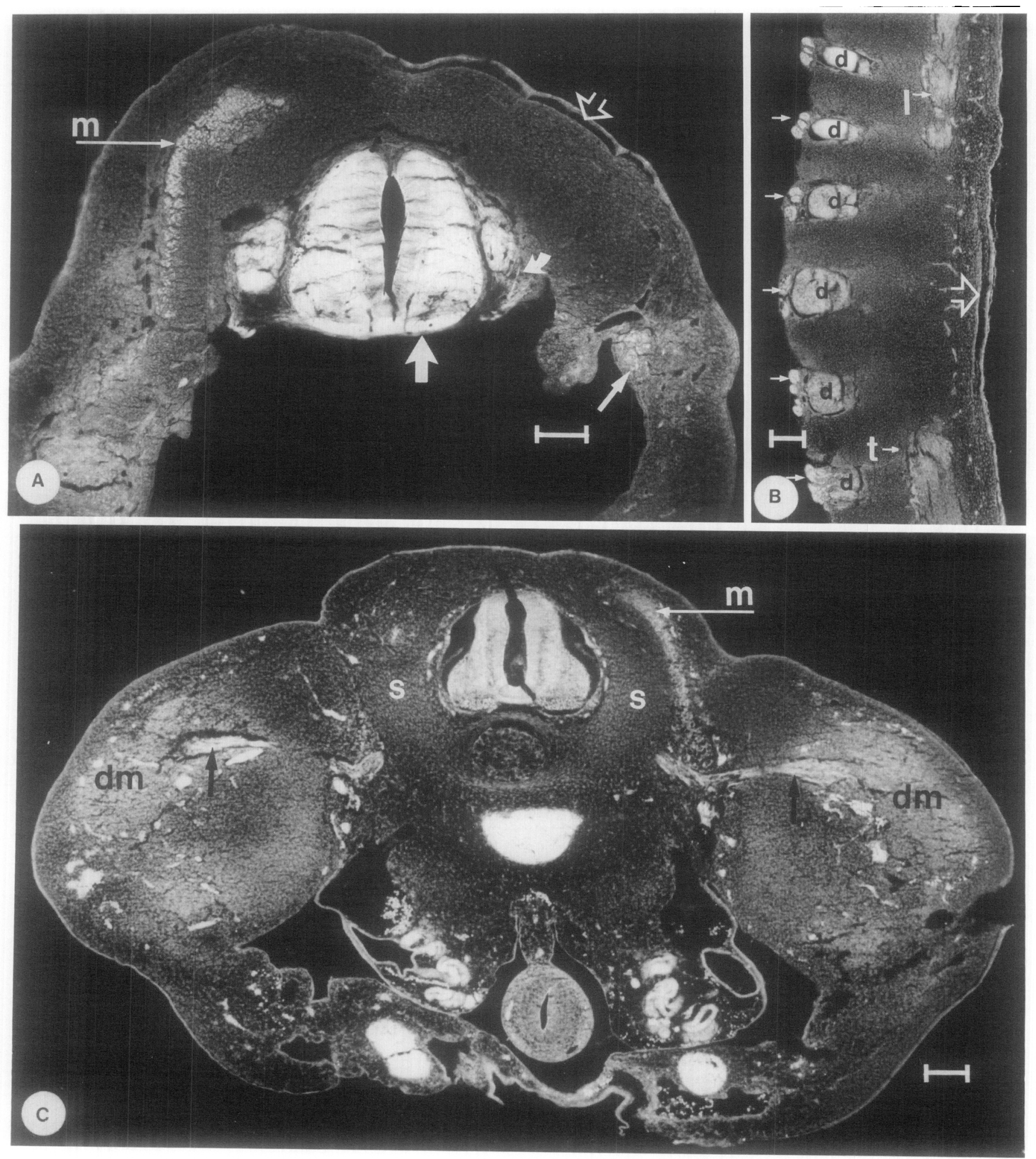

Fic. 2. Embryo development following dermamyotome deletion. (A) A distinet subectodermal space (hollow arrow) indicates the absence of the dermis. Note that the space does not extend past the midline or into lateral trunk regions. The myotome (m) is distinct on the control side and is represented by fewer than 20 myofibers (small arrow) in the thoracic body wall of the experimental side. The DRG on the experimental side appears smaller because the plane of section is slightly oblique. HRP injected into the ventral roots has labeled (among others) cell somata in the epaxial muscle pool (large arrow). A few axons in the dorsal ramus (curved arrow) are visible in epifluorescence. Stage 26 embryo. (B) Lungitudinal section of a stage 26 embryo following removal of demanyotomes from LS1-3 also shows a subectodernal space (hollow arrow) at the operation site. Myotomes are present only at thoracic ( $t$ ) and more posterior lumosacral (1) levels. Despite this, DRG (d) and ventral roots (small arrows) have formed in their normal segmental positions. (C) The myotome ( $m$ on the right) is clearly absent on the operated side (left; the small patch of light cells in this area are red blood cells). The dorsal muscle masses (dm) appear equivalent in size and position on both sides and each is served by a dorsal crural nerve trunk (black arrows). Other tissues, including the sclerotome (s), appear remarkably normal. Three adjacent dermamyotomes had been removed from the crural region. Stage 26 embryo. Epifiuorescence micrographs. Calibration bars, $100 \mu \mathrm{m}$. 

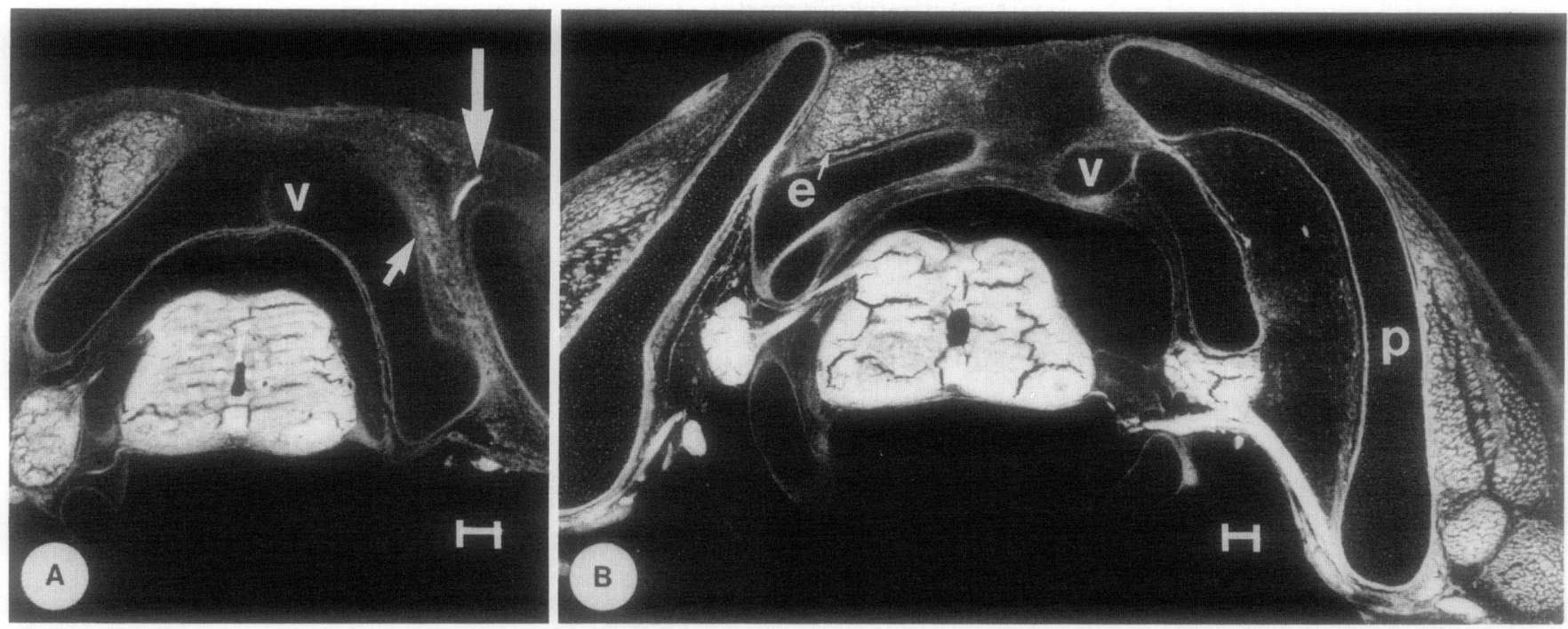

FIG. 3. Later development following dermamyotome deletions. (A) The dorsal vertebrae (v) are nearly normal in conformation in this stage 31 embryo. On the experimental side (right) a few myofibers (small arrow) are seen in a dorsal position between the vertebrae and pelvic girdle. A dorsal ramus grew into this fragment and in this section a cutaneous nerve (large arrow) can be seen projecting dorsolaterally. The cutaneous nerve formed only in cases such as this, when the dorsal ramus had grown to a dorsal position. The DRG are not visible on the operated side because the plane of section is slightly oblique. (B) The epaxial muscle (e) is present on the control side (left) and absent on the experimental side (right) of this stage 34 embryo. Abnormal vertebral condensations (v) and enlarged spaces adjacent to the spinal cord and between the vertebrae and pelvic girdle $(p)$ were occasionally seen in older embryos. Despite these abnormalities, the DRG, spinal nerves, and muscles distal to the pelvic girdle are normal. Epifluorescence micrographs. The ectoderm of each embryo was removed during processing. Calibration bars, $100 \mu \mathrm{m}$.

remove the most lateral portions of thoracie dermamyotomes since these begin to spread distally by late stage 17 and in 7 cases far lateral fragments were left in the embryo. These fragments invariably contributed to intercostal muscles even when the epaxial muscles in the same segment were absent or represented by a small lateral fragment. The loss of proximal dermamyotome apparently does not impede the lateral spread of the remaining tissue into the lateral body walls.

Segmentation of DRG in the absence of the dermamyotome. The segmentation of DRG was not altered when dermamyotomes were partially or totally removed. In every case DRG that formed were found in their normal segmental positions (Figs. $2 \mathrm{~B}$ and 8 ). This shows that the segmental pattern of DRG formation is not dependent on the presence of the dermamyotome.

DRG were absent or smaller than normal in 49 out of 105 operated segments. This observation suggested that the dermamyotome might be essential for the condensation of the neural crest cells that form the DRG. If this were the case, loss of DRG should correlate with complete dermamyotome deletion, depletion of DRG with partial deletion. In addition, if only lateral fragments of dermamyotome remained, the DRG might be expected to condense more lateral than normal, subjacent to the fragment. However, the incidences of normal DRG were similar following complete or partial deletions (Fig. 4) and they were always positioned correctly on the medial-lateral axis.
DRG would also be small or absent if the dermamyotome were essential for migration of their precursors through the somite. However, this possibility is not consistent with the normal size of DRG in 56 out of 105

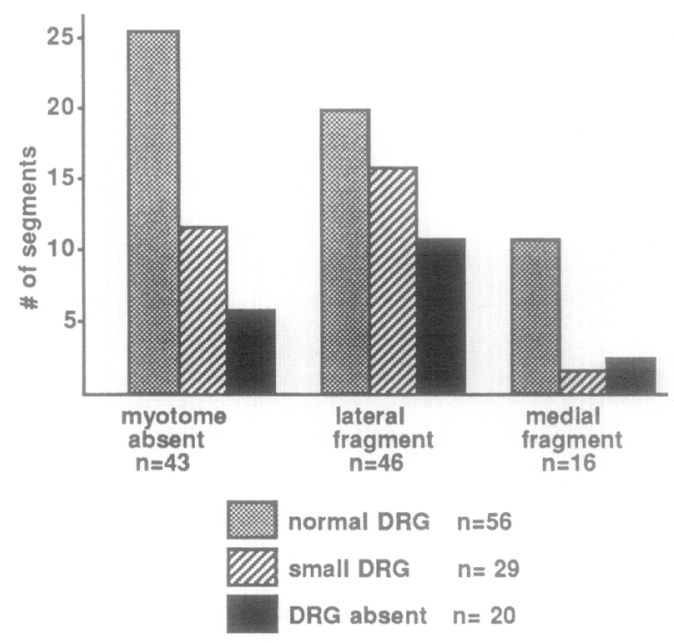

FIG. 4. The relation between the size of the DRG and the degree or position of dermamyotome deletion was examined in 105 operated segments to determine whether condensation of DRG was affected by dermamyotome deletion. A substantial proportion of DRG was normal when the myotome was absent or when only lateral or medial fragments remained. The slight tendency for DRG to be of normal size when a medial fragment remained can be explained by the greater likelihood that removing the medial portion of the dermamyotome also removed adjacent neural crest cells. 
operated segments. It is more likely that depleted DRG resulted from inadvertant removal of neural crest cells. Much of the crest population was mesenchymal during the operated stages and may have been more easily aspirated out than adjacent tissues (like the sclerotome) that were semiepithelial and more coherent. In fact, more tissue had apparantly been removed than desired in some embryos in which the dorsal neural tube or dorsal sclerotome appeared depleted or the subectodermal space extended across the midline. In these cases the proportion of depleted DRG was much greater (Fig. 5). In addition, more DRG were depleted in the more severe operations in which several, rather than a single, dermamyotomes had been deleted (Fig. 5). Direct neural crest depletion is also consistent with a corresponding reduction of DRG on the contralateral side. In $85 \%$ of the cases where DRG were absent and in $52 \%$ of the cases where DRG were small the contralateral DRG were reduced as well (Fig. 6). The contralateral dermamyotome appeared to be undisturbed in all cases. Only $11 \%$ of the embryos displayed a reduction of DRG without either contralateral effects or obvious evidence of a more extensive operation.

If the dermamyotome were important during some critical phase of neural crest migration, then the degree of DRG depletion should correlate with the phase of neural crest migration at the time of the deletion. For instance, when the dermamyotome is removed before neural crest enters the somite, fewer normal DRG should form. However, no relationship was found between the size or presence of DRG and any phase of crest migration

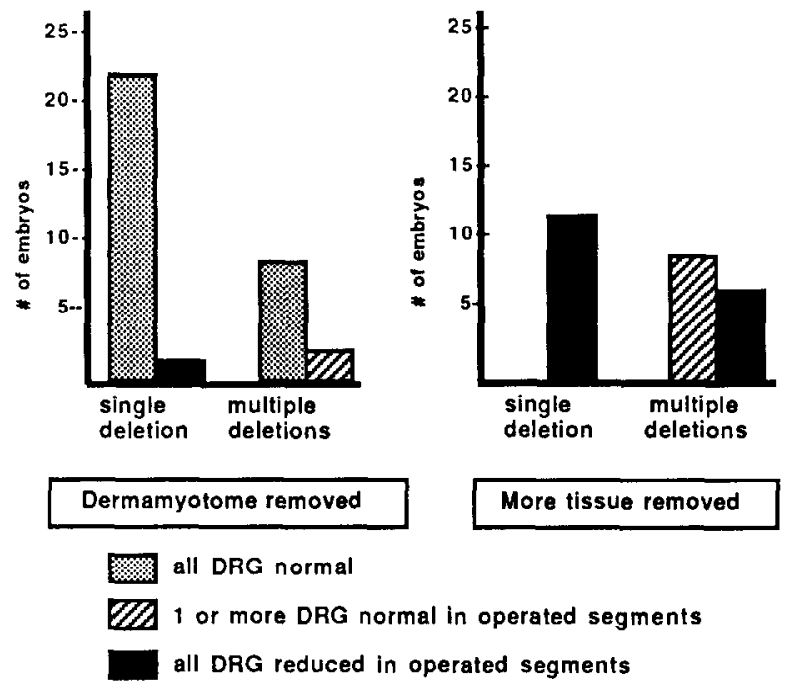

Fig. 5. Following more extensive operations during which neural crest may have been inadvertently removed, the spinal cord or sclerotome was depleted dorsally and the subectodermal space extended across the midline. More embryos had deficient DRG in these cases. In addition, multiple deletions required more extensive surgery than single deletions and resulted in a higher incidence of depleted DRG.

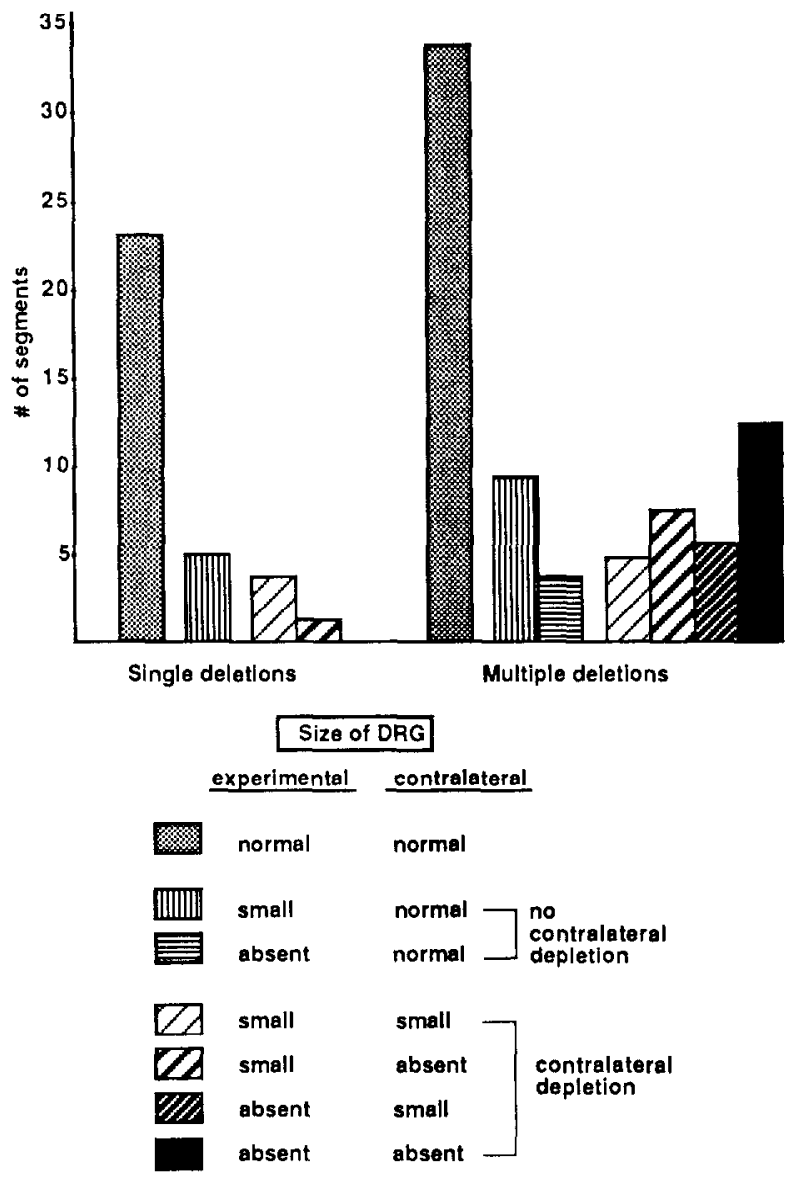

FIG. 6. The size of DRG on experimental and contralateral sides was examined in 105 operated segments following deletions of single or multiple dermamyotomes. Fifty-one segments (53\%) had normal DRG. In the majority of the cases there was a clear correlation between sizes of DRG on contralateral and operated sides. Of the 20 segments in which DRG were absent, DRG were also reduced in 17 contralateral segments (85\%); of the 29 segments where DRG were small, the DRG were also reduced in 15 contralateral segments (52\%). In addition, while more DRG were depleted following multiple deletions, there were also clear contralateral effects following deletions of dermamyotomes in single segments. Since DRG on control and experimental sides often shared the same fate, it is likely that occasional DRG depletion was due to removal of neural crest during the operation rather than to the absence of the dermamyotome.

(Fig. 7). These results show that the neural crest cells that form DRG do not require the dermamyotome for migration.

Segmentation of axon outgrowth in the absence of the dermamyotome. Following single (Fig. $8 \Lambda$ ) or multiple (Figs. 8B, 8C) dermamyotome deletions the gross anatomical nerve pattern was normal in 102/105 segments with the exception of the dorsal ramus (discussed below). For example, in Fig. 8C the second through the sixth lumbosacral dermamyotomes have been removed and the spinal nerves, plexi, nerve trunks, and muscle nerves within the leg are in their normal positions and relationships. This figure also shows that spinal nerves can 


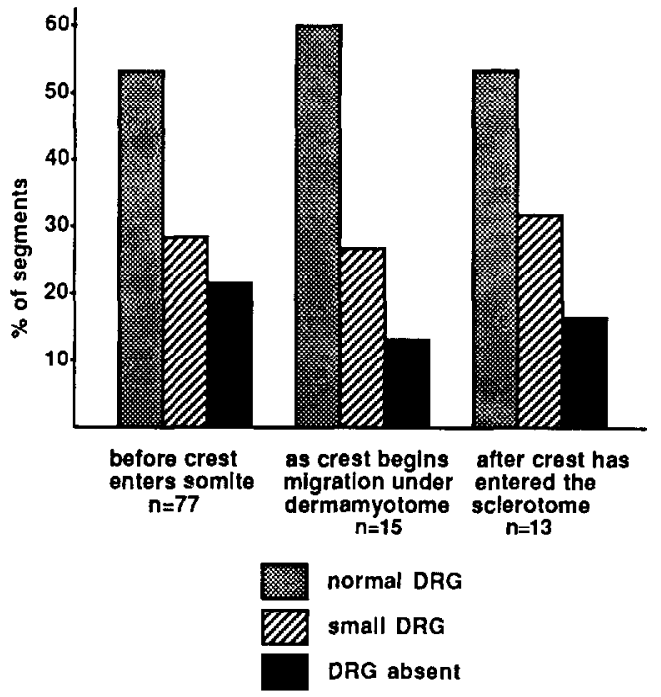

FIG. 7. There was no consistent correlation between the size of DRG in 105 operated segments and the phase of crest migration at the operation site. The proportions of DRG that were of normal size, small, or absent were similar following dermamyotome removal before crest entered the somite (1-6 somites anterior to the segmental plate), as crest began migration under the dermamyotome (7-9 somites anterior to the segmental plate), and after crest had begun to accumulate within the sclerotome.

form normally in the absence of the DRG, confirming the work of others (Lewis et al., 1983; Scott, 1984, Yip, 1987) that motoneuron axons do not require sensory neurites for outgrowth.

Distal outgrowth was abnormal in three additional thoracic segments, all of which displayed multiple irregularities. Dermamyotomal derivatives were absent, the mesenchyme of the sclerotome and the lateral body wall was abnormally compacted, an intercostal nerve did not form, the spinal nerve terminated in a neuroma close to the spinal cord, and DRG were absent on both sides (not shown). The absence of normal outgrowth in these cases may be attributable to extensive neural crest deletion or direct damage to the outgrowth environment.

Spinal nerves in depleted segments were in a few cases $20-50 \%$ shorter than those on the contralateral side (Figs. 9B and 9C). In these cases enough distal tissue had been removed to reduce the distance that axons had to traverse to reach the limb base. Despite the shorter distance they had to travel, their ramification within the limb was always comparable to that of the control side. This observation is of interest because axons normally delay their distal progress into the limb: the first axons reach the plexus region by stage 21 but do not enter the leg until stage 25 (Hollyday, 1983, Tosney and Landmesser, 1985a). The failure of these axons to enter the limb precociously despite their early arrival at the limb base suggests that the intrinsic rate of axon elongation or the process of sorting out in the plexus is not normally responsible for the delay. This observation is in accord with Landmesser's (1987) in vitro evidence that axons wait to enter the limb because the early (but not late) limb mesenchyme is inimical to axon outgrowth. The result suggests further that it is not the growth cones that alter the distal environment and render it suitable for advancement.

In order to determine whether the dorsal-ventral organization of spinal nerves was abnormal, 30 embryos were closely examined at stages $21-25$ when most growth cones are still within the proximal environment. In most of these the spinal cord or ventral root had been injected with HRP to enhance the probability of finding errant axons. In all cases the emerging spinal nerves were as coherent as they normally are and errant axons were not seen. In those cases where three or more contiguous dermamyotomes had been completely removed, spinal nerves were exceptionally coherent since (as described below) growth cones did not emerge from the dorsal surface of the spinal nerve to form the dorsal ramus in the central deprived segments. The relative coherence of such spinal nerves is shown in Fig. 10. Excluding the three exceptions discussed above, orderly spinal nerve outgrowth was characteristic of all embryos, even those operated upon at the earliest and latest stages, regardless of the degrec or position of dermamyotome deletion.

It is possible that the dermamyotome normally provides navigational cues but that, in its absence, axons can utilize secondary cues from other sources. If this were the case, imprecisions in outgrowth might be detectable. For instance, axons might wander more and show convoluted trajectories within the spinal nerve pathway. This was clearly not the case: axons within the spinal nerve pursued relatively straight trajectories even at early stages of outgrowth (Fig. 9). The degree of fiber order was similar to that seen in unoperated embryos or on the contralateral side.

These observations show that the dermamyotome is not required for the distal outgrowth of axons to the leg base or for normal spinal nerve segmentation and coherence on the dorsal-ventral axis.

Specificity of motoneuron projection into the limb following dermamyotome deletion. Leg muscles were innervated with normal specificity from pools in both deprived and adjacent segments following removal of single ( $N=16$ embryos) or multiple ( $N=15$ embryos) dermamyotomes. For example, in Fig. 8A, the dermamyotome had been removed at thoracic level 7. Axons project correctly from motoneurons in the adjacent segments LS1 and LS2 to the sartorius muscle and from sensory neurons in the first and second lumbosacral DRG into the sartorius and anterior cutaneous nerves. Axons were not labeled in the depleted segment anterior to this pool, showing that there was no detectable shift in projection in the absence of the dermamyotome. Similarly, in Figs. 8B and 8C axons project correctly through 

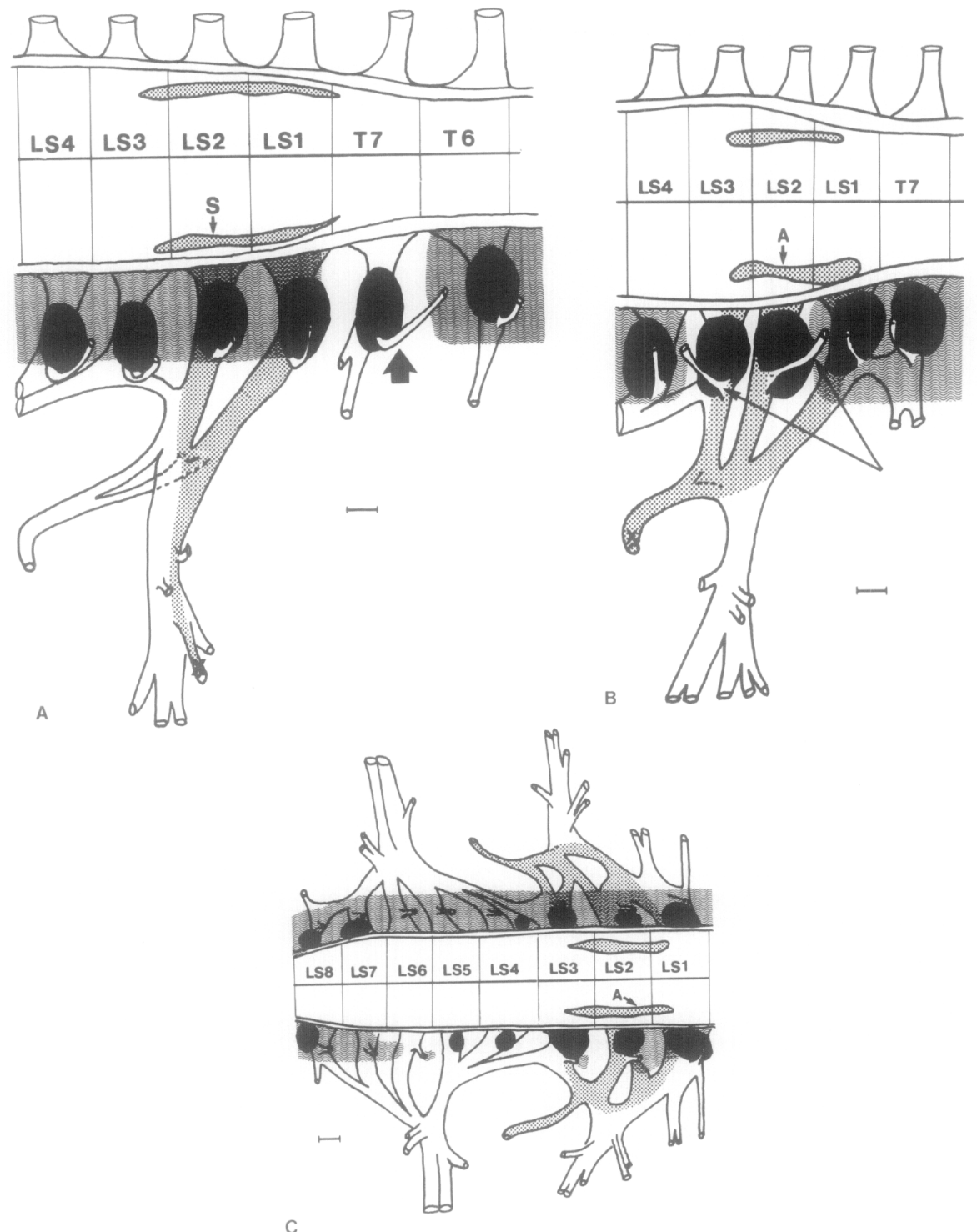

Fig. 8. Reconstructions of the nerve patterns following dermamyotome deletions. The experimental side is to the bottom in each figure and experimental and contralateral sides received similar HRP injections. (A) and (B) show only the anterior lumbosacral region; the posterior was normal. Stippling represents HRP label, wavy lines the epaxial muscles, and dark stippling the DRG. Only the DRG in the appropriate segments had labeled somata. The segment boundaries within the spinal cord have been arbitrarily drawn between DRG, using the criteria of Landmesser (1978). Segments do not correspond to the former somite boundaries because tissues shifted during development (cf. Beresford, 1983) so that the spinal nerve appears to lie toward the middle to posterior of each segment in what was formerly the anterior portion of the somite. Segmentation of outgrowth was comparable on control and operated sides in all embryos. (A) Following complete deletion of a single dermamyotome the dorsal ramus (arrow) ends in epaxial muscle in the more anterior segment. During initial outgrowth, this would have been the closest epaxial muscle target. The segmentation of DRG and spinal nerves as well as the gross anatomical nerve pattern in the limb are normal. The sartorius muscle nerve was injected on both sides and labeled somata (s) lie in their normal position within the spinal cord in both anterior-posterior and medial-lateral planes. Stage 31 embryo. (B) Two adjacent dermamyotomes have been completely deleted and each dorsal ramus (arrows) ends in epaxial muscle in the closest adjacent segment. The segmentation of spinal nerves and DRG is normal, as is the gross anatomical nerve pattern in the limb. Adductor muscles are innervated from motoneurons in the normal position in the spinal cord (A). Stage 31 embryo. (C) Dorsal rami are absent in segments LS4 and LS5, where dermamyotomes have been completely deleted and adjacent dermamyotomes have been severely depleted. Dorsal rami in segments 3 and 6 end in small epaxial muscle fragments. Spinal nerve segmentation is normal, but DRG are depleted on both sides in the posterior. Note that the dorsal ramus can form in the absence of $\mathrm{DKG}$. Motoneurons innervating the adductor muscles (A) lie in their normal position in the spinal cord. Stage 27 embryo. Calibration bars, $100 \mu \mathrm{m}$.

deprived segments to the ventral nerve trunk. Motoneuron pools labeled from peripheral injections lay in their normal anterior posterior and medial-lateral positions within the spinal cord (Figs. 8 and 11). Injections into the spinal cord, ventral roots, or spinal nerve allowed the precision of innervation of several limb muscles to 

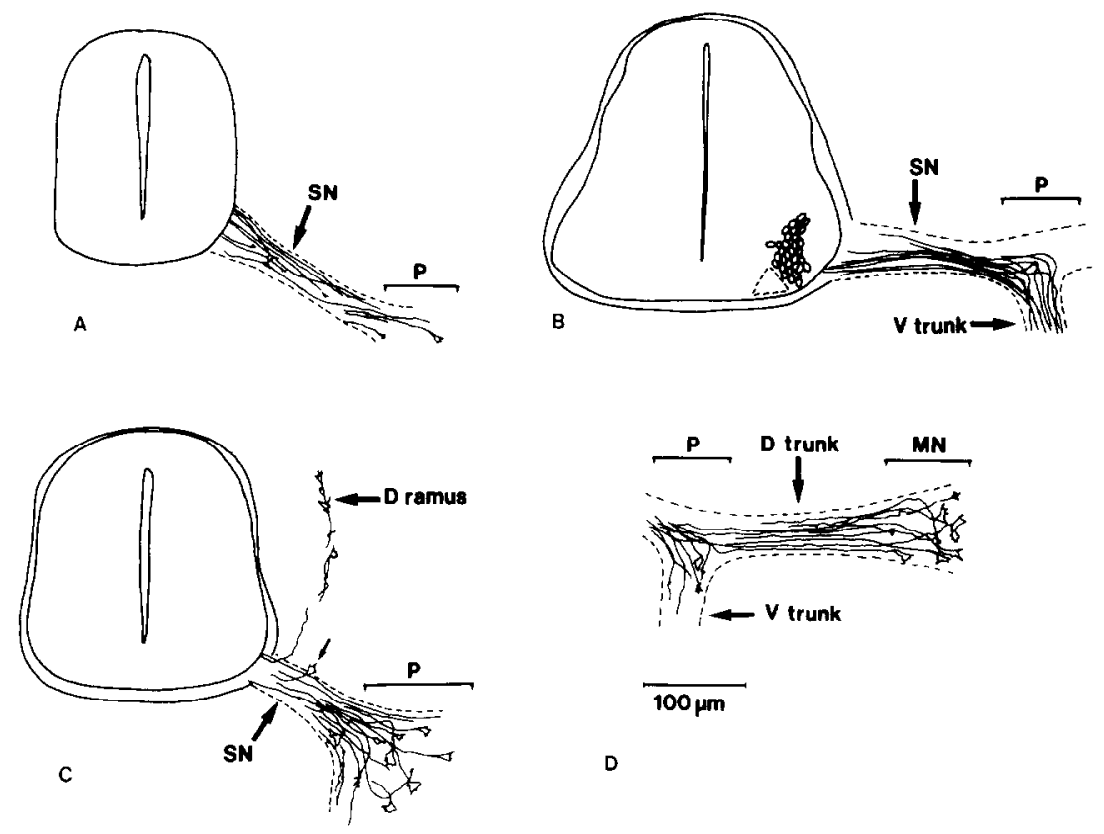

FIG. 9. Axon trajectory following dermamyotome deletions. HRP was injecled into the spinal cord (A, C, and D) or the ventral nerve trunk (B), and labeled axons with trajectories sufficiently long to be followed within one section were traced with a camera lucida. Each figure is a composite of 3-5 semiserial sections. In normal embryos, axons pursue straight trajectories and end in small growth cones in the spinal nerve and nerve trunks; in contrast, they alter their trajectories and often end in enlarged growth cones in the plexus region $(\mathrm{P})$ and regions of incipient muscle nerve formation (MN). This position-dependent pattern is also found in the experimental embryos. In (A), a stage 22 embryo, motoneuron axons pursue a relatively straight trajectory and do not ramify beyond the boundaries of the spinal nerve (SN). A single dermamyotome had been completely removed but the injection was small and while axons in the incipient dorsal ramus were visible with epifluorescence, they were not labeled. Three adjacent dermamyotomes had been removed from the stage 27 embryo shown in (B). A dorsal ramus was not detectable. The distal HRP injection into the ventral nerve trunk labeled only somata (circles) that lie in the normal position for motoneurons that project through that trunk; motoneurons within the dorsal ramus pool (outlined by dotted lines) are not labeled. Axons run roughly parallel in the spinal nerves and ventral nerve trunk and alter their trajectory in the plexus region. The spinal nerve was approximately $20 \%$ shorter than that on the control side. In (C), a stage 24 embryo, the spinal nerve (SN) was approximately $50 \%$ shorter than normal, but the nerve plexus (P) had formed in its normal position relative to limb tissues and axons had progressed into the limb for equivalent distances on both sides. Axons in the spinal nerve are roughly parallel, but drastically alter their trajectories in the plexus region, where large growth cones are also seen. Labeled axons and growth cones are present in the incipient dorsal ramus (D ramus) in the absence of a single dermamyotome. A growth cone exiting from the spinal nerve (small arrow) may be a member of the same population. (D) Plexus region (P), nerve trunks, and a region of incipient muscle nerve formation (MN) from a stage 26 embryo. Again, axon trajectories are roughly parallel in the nerve trunks and enlarged growth cones are seen in the "decision regions."

be examined in each embryo and in all cases this segmental pattern of projection was normal $(N=14 \mathrm{em}-$ bryos). These observations show that target selection in the limb is unaltered in the face of extensive dermamyotome deletion.

Since axons can select the correct target using cues within the limb even when they have made proximal decisions incorrectly (Lance-Jones and Landmesser, $1981 \mathrm{~b}$ ), the finding that muscle innervation is substantially correct does not thoroughly test the hypothesis that the dermamyotome provides information used in pathway selection at the limb base. To determine whether axons had made incorrect choices in the plexus region and had innervated selectively by making distal corrections within the limb, the precision of dorsal and ventral nerve trunk selection was examined in detail. The ventral nerve trunk was injected with HRP at stage 26 (shortly after its formation) in three embryos with single dermamyotome deletions and in seven embryos with multiple dermamyotome deletions within the crural region. In all cases examination of the position of labeled somata and tracing of axon trajectories showed that the labeled axons had chosen the correct pathway at the limb base (see Fig. 8C). In addition, sorting out along the dorsal-ventral axis was confined to the plexus region as it normally is (Fig. 9B). In particular, axons did not move into the limb and make U-turns, as has been seen in a few instances when it is suspected that the initial precision of sorting out has been impaired by experimental manipulations (see Tosney, 1986, 1987b). Finally, "novel nerves" that are seen only when axons make pathway corrections within the limb (Lance-Jones and Landmesser, 1981a; Lance-Jones, 1986) were not detected in these embryos.

During normal development, motoneuron axons that enter the limb alter their trajectories and exhibit en- 

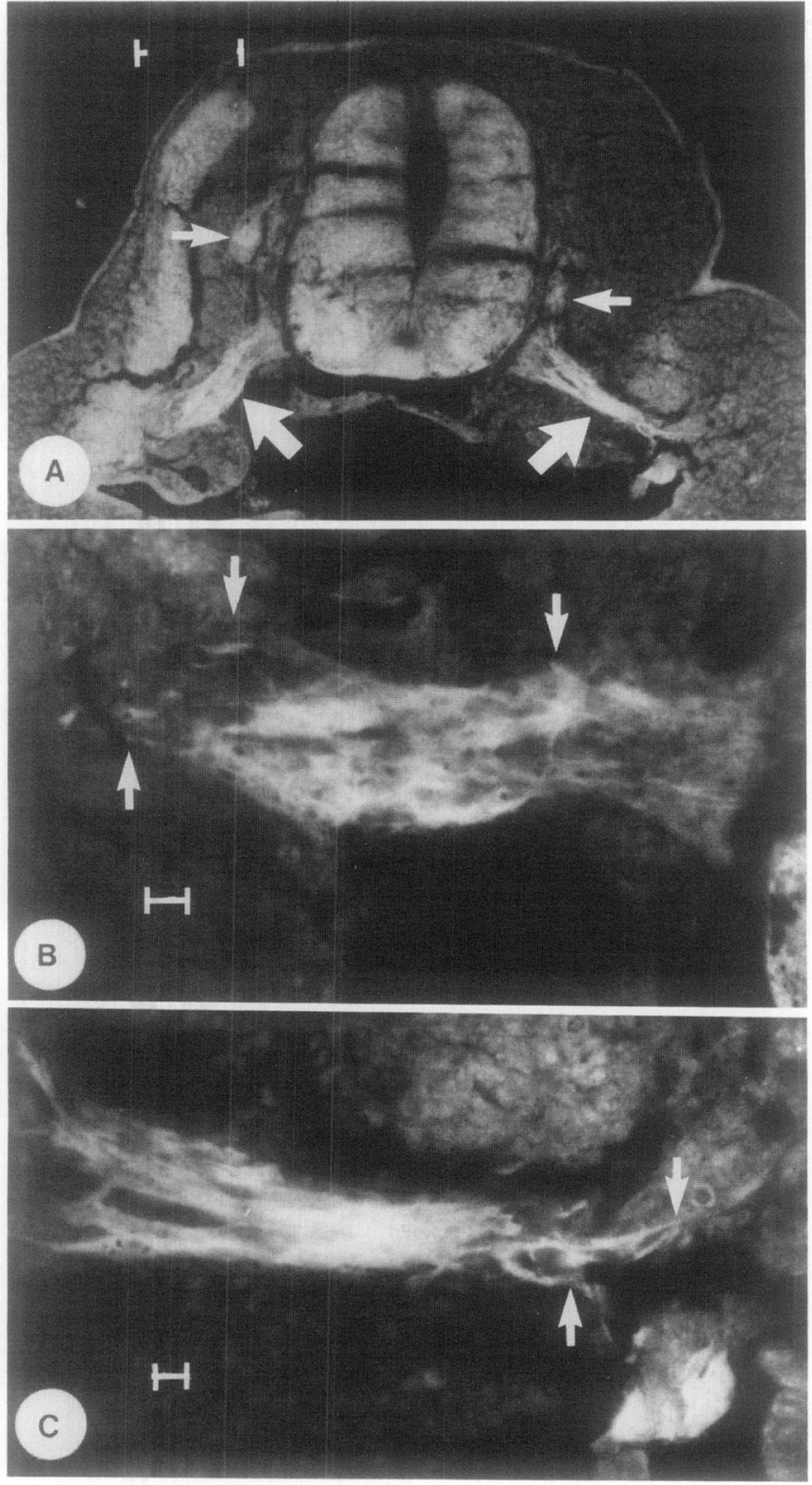

Frg. 10. Spinal nerve equivalence on control and operated sides in the absence of the dermamyotome and the dorsal ramus. (A) Three dermamyotomes have been deleted from this stage 22 embryo and the cross section shown is through the segment in which a dorsal ramus is absent. DRG (small arrows) are beginning to condense lateral to the spinal cord and spinal nerves (large arrows) have progressed to similar positions on each side. The spinal nerve on the control side, shown at higher magnification in (B), has a normal degree of coherence and is composed of fasciculated groups of axons. A few axons (small arrows) fray out from the dorsal surface and the growing nerve front as it approaches the plexus region. On the experimental side, (C), axons (small arrows) are fraying out at the outgrowing tip but the proximal spinal nerve is unusually coherent: axons have not extended dorsally in this or adjacent sections and the fascicles are closely packed. Calibration bars: (A) $100 \mu \mathrm{m}$; (B, C) $10 \mu \mathrm{m}$.

larged growth cones predominantly in "decision regions" (such as the plexus region and areas of muscle nerve initiation) where possible pathways diverge. This elab- oration of growth cones has been suggested to reflect their responses to specific local cues (Tosney and Landmesser, 1985c). To determine whether these positiondependent attributes were altered in operated embryos, axon trajectories were traced following injection of HRP into the spinal cord (stages 21-25) or a nerve trunk (stages 25-26). Figure 9 shows that these attributes were preserved: axons pursued straight trajectories and growth cones were small in spinal nerves and nerve trunks, while axons often altered trajectories and enlarged growth cones were seen in decision regions. Thus, sorting out along the dorsal-ventral axis was normal in all respects, right down to the growth cone level. I conclude that the continued presence of the dermamyotome is not required for accurate pathway selection at the base of the limb.

The dorsal ramus in the absence of its target. The dorsal ramus normally diverges dorsally from each spinal nerve to innervate epaxial muscle and dermis that are derived in situ from each dermamyotome (Tosney, 1987a).

The formation of the dorsal ramus was found to depend on the proximity of the dermamyotome. When a single dermamyotome had been completely removed a dorsal ramus arose from the spinal nerve of the deprived segment and projected dorsally in a normal manner $(N$ $=13$; Fig. $11 \mathrm{~A}$ ). By stage 26 the tip of the dorsal ramus was dorsal to the bulk of the sclerotome in a position where it normally forms terminal branches at this stage. It then turned toward the anterior and extended into epaxial muscle in the adjacent segment $(N=5$; Figs. $11 \mathrm{~B}, 11 \mathrm{C}$, and $8 \mathrm{~A}$ ). It is of particular interest that the nerve extended toward epaxial muscle that was anterior rather than posterior to its normal segment: since the dorsal ramus and the spinal nerve form within the anterior portion of the somite, the epaxial muscle in the next anterior segment is always the closest target.

This preference for the closest epaxial muscle was consistently seen. When two adjacent dermamyotomes were removed and the deletion was complete, the dorsal rami grew dorsally and then along the anterior-posterior axis to enter the nearest epaxial muscle: the anterior ramus extended anteriorly, the posterior extended posteriorly ( $N=3$, sec Fig. 8B). Following multiple deletions, the dorsal rami at the most anterior and posterior deleted segments extended anteriorly and posteriorly, respectively ( $N=10$ embryos), unless a small piece of target tissue remained in their segment. Although the growth of dorsal rami into adjacent segments resulted in apparant dual innervation of epaxial muscles in those segments, neither the "foreign" nor the segmentally correct nerve was seen to retract or was noticeably depleted in size. However, the oldest embryo examined was well before the peak of cell death in the lateral motor column and it is not known whether the dorsal ramus population exhibits target-dependent cell death. 

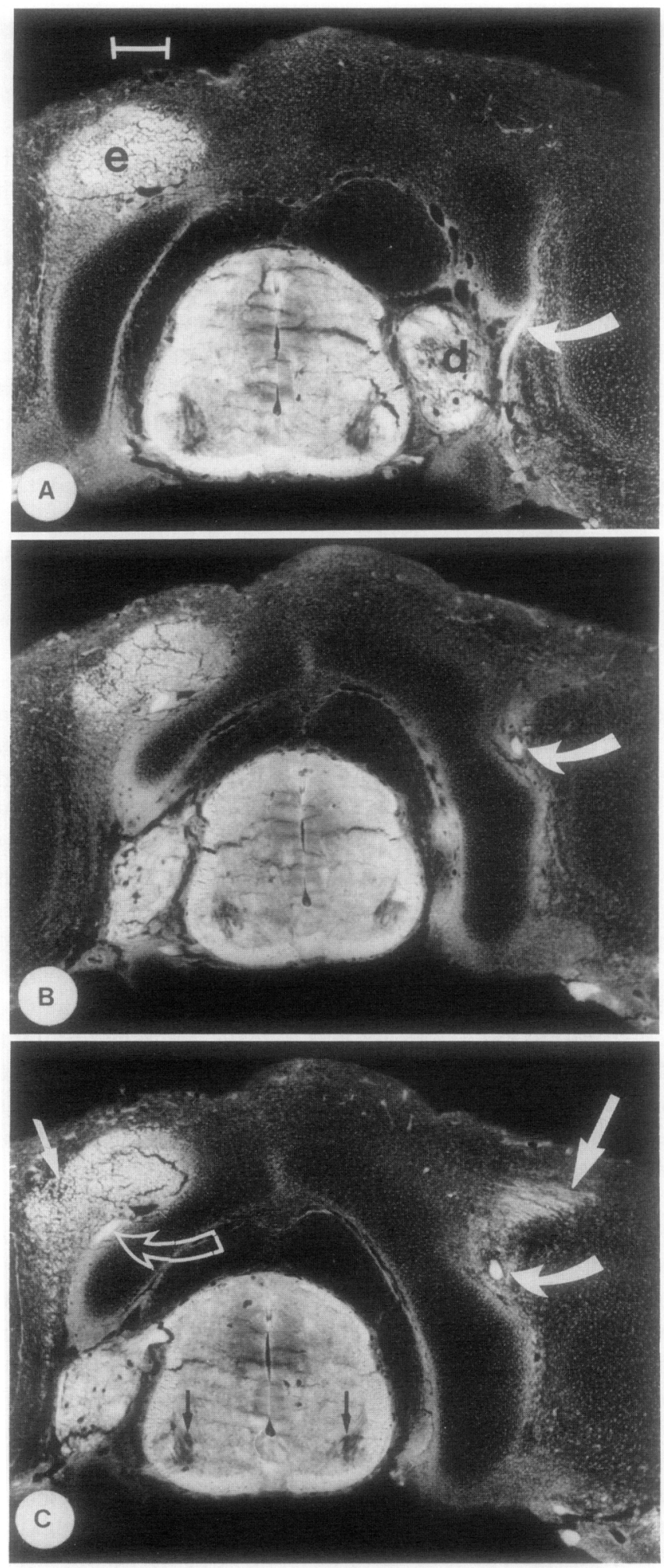

FIr. 11. Projection of the dorsal ramus to the adjacent anterior segment following removal of a single dermamyotome. (A) A dorsal ramus (curved arrow) has projected dorsally in a normal manner despite the absence of the epaxial muscle (e) on the experimental side (right). Note that a normal DRG (d) has formed. (B) In a section 100 $\mu \mathrm{m}$ to the anterior, the dorsal ramus (curved arrow) is seen in cross section as it progresses toward the anterior. The nerve is traveling
When several dermamyotomes were removed the results were dramatically different. A dorsal ramus was absent when a dermamyotome had been completely removed and the dermamyotomes in both adjacent segments had been substantially reduced ( $N=5$ embryos) or were absent ( $N=5$ embryos). For instance, in the reconstruction shown in Fig. $8 \mathrm{C}$ dorsal rami are absent in segments 4 and 5 . Even in the youngest embryos observed (stage 21), axons were never detected exiting from the spinal nerve in these cases, either under fluorescence optics in which very small groups of axons and occasional individuals can be detected (compare Figs. 2A and 10) or following injection of HRP into a spinal cord segment or ventral root (see Fig. 9). Thus it seems highly unlikely that axons leave the spinal nerve in the absence of nearby target and later regress.

When even a small remnant of dermamyotome remained, a dorsal ramus arose in the depleted segment and extended toward (in the youngest embryos) or ended in the fragment ( $N=62$; see Fig. $8 \mathrm{C}$ ). Despite the small size of the target in these cases (the smallest remnants were composed of a dozen or so muscle fibers; see Fig. $3 \mathrm{~A}$ ), axons did not ramify toward the adjacent segments even in embryos as late as stage 34 .

The results strongly suggest that the muscle derivative of the dermamyotome is essential for outgrowth. Fragments that elicited dorsal ramus outgrowth always contained muscle cells (see Fig. 3A; $N=62$ ). When the dorsal ramus was absent, muscle cells were not detectable, even in five segments in which dermis was apparantly present. However, it should be noted that dermis, unlike muscle, can not be identified by cell morphology: dermis and sclerotome cells look similar at these stages. It therefore remains possible that dermis as well as muscle is important for outgrowth.

These observations raise the question, "What is the fate of the dorsal ramus axons in the absence of their target?" If this population were to project to the limb in the absence of their target, it is likely that they would project randomly or in accord with the adjacent moto-

dorsal to the bulk of the sclerotome, in the position where it normally branches to form muscle and cutaneous nerves. (C) Thirty $\mu \mathrm{m}$ more anterior, the dorsal ramus (curved arrow) approaches epaxial muscle in the more anterior segment. The myofibrils (straight arrow) in this muscle are arranged in an abnormal medial-lateral orientation, as though turning about on themselves, while myofibrils on the control side (small arrows) are normally oriented and are cut in cross section. In a more anterior section (not shown) the dorsal ramus moved into the epaxial muscle and a cutaneous nerve formed. The dorsal ramus on the control side (hollow arrow) curves out of the plane of section and its tip moves posteriorly within the muscle and can be seen in (B). The labeled somata (black arrows) in the lateral motor column are in an appropriate position for HRP injected in to the ventral nerve trunk. DRG are also appropriately labeled. Epifluorescence micrographs. Calibration bar, $100 \mu \mathrm{m}$. 
neuron population that enters the ventral nerve trunk. In either case they should have been detected by ventral nerve trunk injections and were not. For instance, in four embryos in which dorsal rami were absent in one or more crural segments, injections into the ventral nerve trunk normally served by these segments did not label somata of epaxial motoneurons (Fig. 10B). I cannot exclude the possibility that the epaxial motoneurons exit the spinal cord and remain in the spinal nerves or nerve plexus. I injected ventral roots in nine embryos following multiple deletions. Somata and axons of epaxial motoneurons were labeled in seven embryos in which a dorsal ramus had grown either to a tiny fragment $(N=3)$ or to an adjacent segment. In one embryo the dorsal ramus formed but neither its axons nor its somata were labeled by the injection. This is not surprising since injections label only a subpopulation of the axons present (see Tosney and Landmesser, 1986) and axons in the dorsal ramus are not labeled by similar injections in $20 \%$ of unoperated embryos. In the remaining embryo a dorsal ramus was absent and epaxial motoneurons were not labeled by the ventral root injection. This single case suggests that axons may not leave the spinal cord in the absence of their target. However, because dorsal rami are not labeled by every injection, the evidence is by no means compelling.

The culantous corrponent of the dorsal ramus. The dorsal ramus is a mixed nerve with both sensory and motor components. The muscle nerves have sensory afferents and a cutaneous nerve emerges from the dorsal ramus just ventral to the epaxial muscle nerve branches and projects dorsolaterally toward the ectoderm (Tosney, 1987a). All elements were absent when three or more contiguous dermamyotomes had been completely removed. The absence of sensory afferents is in accord with the hypothesis of Honig (1982) that sensory axons require motoneuron axons in order to innervate muscle targets. It is also possible that the dermis, the ectoderm, or the muscle provides a chemotactic cue for these sensory neurons. Indeed, the outgrowth of sensory axons in culture can be influenced by coculture with some of these targets (Lumsden and Davies, 1983; Verna, 1985; Verna et al., 1986).

The present results suggest that chemotaxis to the prospective target is not a sufficient mechanism for outgrowth of these cutaneous axons. For instance, in five embryos in which myotomes were absent, a substantial amount of dermis appeared to be present. If the dermis were sufficient to elicit outgrowth of cutaneous afferents, I would have expected to see a dorsal ramus (composed only of sensory fibers) emerging in these, and only these, cases of multiple dermamyotome removal. This was not the case. Clearly, the ectoderm does not provide a sufficient cue since it was substantially undisturbed in the majority of the embryos. If the ectoderm or the dermis does attract these axons, its attraction is insufficient to elict outgrowth from the distant DRG.

Likewise, epaxial muscle and motor axons within the dorsal ramus are not, by themselves, sufficient for outgrowth of these cutaneous axons since the cutaneous nerve did not form in all operated segments in which these were present: it formed only when the dorsal ramus had grown dorsally. For instance, in seven stage 30-34 embryos in which a cutaneous branch was present the dorsal ramus had grown dorsally, to reach either a dorsal muscle fragment within that segment (Fig. 3A) or a muscle in an adjacent segment. Two additional embryos within this age class did not have a cutaneous nerve. In these the dorsal ramus grew dorsolaterally and ended entirely within a ventral muscle fragment. In these two cases, cutaneous axons in the ramus may not have come close enough to the ectoderm and dermis for a possible chemotactic attraction. The ventrolateral trajectory of the dorsal ramus also precludes any direct interaction between motor axons in the ramus and the sensory ganglion. The ramus normally forms adjacent to the DRG (Tosney, 1987a) and it is possible that its proximity is required for the egress of these sensory axons from the ganglion. Blood vessels may also provide essential substrata for outgrowth since the cutaneous branch normally forms in association with a blood vessel (Tosney, 1987a) and a blood vessel did not approach the ectoderm from the ventral muscle fragment in either embryo. A final possibility is that dorsal but not ventral epaxial muscle attracts the cutancous axons.

The cutaneous branch in these two embryos could have regressed in the absence of appropriate target. To determine whether this were the case, 19 operated segments containing DRG of normal size were examined at stage 26 , since the cutaneous branch is normally first detectable during the later half of this stage. In 7 of these segments the cutaneous branch had formed on the contralateral side. The cutaneous branch had formed on the operated side in only 2 of these segments; in both cases the dorsal ramus had extended into dorsal muscle fragments. The cutaneous branch was not detectable on the experimental side in the remaining 5 segments. In 4 of these the removal of dermis was extensive. However, in all 5 the dorsal ramus extended ventrolaterally toward a ventral muscle fragment and a blood vessel did not extend from the fragment toward the ectoderm. Therefore, the formation of the cutaneous branch correlates with the projection of the ramus to a dorsal position during its initial formation as well as at later stages.

These observations suggest that a dual set of outgrowth cues is important for proper formation of the cutaneous branch of the dorsal ramus: a set of chemotactic cues or preferred substrata to guide these growth 
cones dorsally and further chemotactic cues or preferred substrata for the formation of the cutaneous branch itself.

While these observations are consistent with the possibility that the outgrowth of sensory axons somchow depends on outgrowth of motoneuron axons, the converse is not true: an anatomically correct dorsal ramus can form to muscle targets in the complete absence of the DRG (Fig. 8C). This shows that dorsal ramus motoneurons do not require sensory axons for outgrowth.

\section{DISCUSSION}

Tissues between the spinal cord and limb base appear to provide specific cues as well as permissive pathways for axonal outgrowth. For instance, proximal tissues are sufficient for remarkably faithful pathway choice at the base of the limb (Tosney and Landmesser, 1984; LanceJones, 1986; Phelan and Hollyday, 1986) and preganglionic (Yip, 1987) and dorsal ramus (Tosney, 1987a) axons project with fidelity down divergent pathways within the somite. Recent attempts to determine the roles of various proximal tissues have identified some elements that delincate permissive pathways. For instance, the developing pelvic girdle at the limb base is a barrier to axonal advancement (Tosney and Landmesser, 1984) and the segmental pattern of both axon (Keynes and Stern, 1984) and neural crest outgrowth (Rickman et al., 1985; Loring and Erickson, 1987; Newgreen et al., 1986) is due to differences of an unknown nature in anterior versus posterior somitic tissue.

I refined the analysis of the role of somitic tissues by deleting the histologically distinct dorsal portion of the somite, the dermamyotome, and examining subsequent development. It was surprising to find that, with the exception of dorsal ramus formation (diseussed below), none of the developmental events suspected to depend on the dermamyotome were altered by its deletion. Neural crest cells that form sensory ganglia migrated and condensed in their normal segmental pattern. Spinal nerves were organized normally on anterior-posterior and dorsal-ventral axes and their distal progression was not disturbed. In addition, motoneuron axons sorted out correctly in all respects in the plexus region and projected to their normal targets in the limb. Since axon outgrowth is likely to require a balance among multiple mechanisms and since deletion strategies can only tell us if a tissue is essential, it is still possible that the dermamyotome contributes in some replaceable manner to patterned outgrowth. Other experimental designs must be used to assess this possibility.

Target proximity and the dorsal ramus. During normal development, dorsal ramus axons emerge with other motor axons in the ventral roots. The motor axons destined to innervate distant limb targets remain in the spinal nerves and make a number of complex pathway decisions as they traverse the distal environment. In contrast, the dorsal ramus axons are close to their target. They diverge quickly from the spinal nerve and procede directly to their destination (sce Tosncy, 1987a). Onc important issue addressed in this study is to what extent these two motoneuron populations utilize different arrays of cues to attain their proper destinations. Do the dorsal ramus axons respond specifically to their nearby target?

The answer to this question is yes, the outgrowth of dorsal ramus motoneurons is clearly dependent on the proximity of the dermamyotome. When a single or two adjacent dermamyotomes were removed, the corresponding epaxial muscles were absent and the dorsal ramus entered epaxial muscle in the closest adjacent segment. However, the dorsal ramus did not form when dermamyotomes in the contiguous segments had also been removed or substantially reduced. Since these axons are readily identifiable with epifluorescence or HRP labeling, it is unlikely that the growth cones grew out undetected and then regressed in the absence of their target.

The motoneuron axons of the dorsal ramus appear to respond to the myotome (and muscle) rather than to the dermatome (and dermal) derivatives of the dermamyotome. The correlation between outgrowth and the presence of even a few muscle fibers in a segment was clear and consistent. In contrast, in a few cases dermis appeared to be present without eliciting outgrowth. However, because the identification of dermis (by position) is less certain than the identification of myotomal derivatives, I could not conclusively determine whether dermis as well as muscle is essential for outgrowth.

The observations strongly suggest that the dorsal ramus axons require a chemotactic signal for outgrowth. During normal development, the dermamyotome is 80$100 \mu \mathrm{m}$ from the spinal cord, beyond the filipodial grasp of growth cones emerging in the ventral roots (Tosney, 1987a). In addition, this target appears to be able to elicit directed outgrowth up to $300 \mu \mathrm{m}$ away (the approximate distance between a spinal nerve and the dermamyotome in the more posterior segment) and is ineffective beyond $600 \mu \mathrm{m}$. This distance is within the acceptable range for diffusion of a chemotactic molecule in vivo (see Crick, 1970). Recently, diffusible chemotactic cues from Xenopus myotomes have been elegantly demonstrated by McCaig (1986), suggesting that the outgrowth of motoneurons to these nearby targets may be chemotactic in a number of organisms. It is not known whether these cues act in soluble form or by binding to cells or extracellular matrix and providing a substratumattached gradient of adhesiveness.

Dorsal ramus motor axons were never detected within 
the limb in the absence of nearby dermamyotome. This suggests that the limb muscle targets are too distant or are inappropriate for effective attraction. However, it could not be conclusively determined whether axons enter the spinal nerves in the absence of their epaxial muscle targets. The cue provided by the dermamyotome could thus be essential either for divergence of the axons from the spinal nerve or for their outgrowth from the spinal cord itself.

It was previously proposed that the growth cones from this population (earlier termed the "myotomal" growth cones) may respond to the proximity of their target (Tosney and Landmesser, 1985c). During their initial outgrowth, these growth cones are unusually large and elaborate and appear similar in these respects to growth cones that are within regions where they must discriminate between alternative pathways to limb targets (Tosney and Landmesser, 1985c). Similar position-dependent variations in growth cone morphology have been described in other systems (Taghert et al., 1982; Roberts and Taylor, 1983; Bentley and Caudy, 1984; Bovolenta and Mason, 1985; Caudy and Bentley, 1986). The fact that the growth cones of dorsal ramus motoneurons are indeed dependent on their target for outgrowth lends credence to the proposal that variations in growth cone size and form are in some real sense responses to specific cues.

Limb motoneurons have been suggested to respond to two types of target attraction: a general attraction that may be due to myoblast maturity and a specific attraction that correlates with target identity (cf. Lance-Jones and Landmesser, 1981a,b; Lance-Jones, 1986, 1987). General target attraction may elicit the formation of muscle nerves in the limb since these are absent when muscle is absent (Lewis et al., 1981) but form in their normal positions even when innervation is nonselective or forcign (Lance-Jones and Landmesser, 1981a). Muscle nerves form at sites within the muscle masses where myotubes first mature (cf. Noakes et al., 1986). For instance, the myotubes at these sites exhibit the earliest immunoreactivity to the neural cell adhesion molecule (NCAM) within the muscle masses, and the ramification of axons within developing muscles progresses in concert with an increase in NCAM immunoreactivity on myotube surfaces (Tosney et al., 1986).

The dorsal ramus may also respond to a general signal of myotube maturity. Axons are first detectable in the dorsal ramus soon after the first myotubes form and display NCAM immunoreactivity (Tosney, 1987a, Tosney et $a l,, 1986)$. It is thus possible that maturing myotubes attract dorsal ramus axons. This is consistent with the finding that spinal cord neurites in Xenopus are attracted by somitic myoblast cells but not hy their undifferentiated precursors (McCaig, 1986). However, since limb motoneurons do not respond in any detectable manner to the dermamyotome, the effective signal would have to differ in epaxial and limb myotubes. Alternatively, limb motoneurons may gain an ability to respond to target cues as they enter the limb. It would be interesting to determine whether dorsal ramus axons would respond to limb muscle if it were placed within chemotactic range.

In the limb, specific target attraction may explain the ability of axons to form novel paths to their correct targets when they have been channeled into foreign pathways (Lance-Jones and Landmesser, 1981a; Lance-Jones, $1986 \mathrm{~b})$. Although these novel nerves form at the same time as normal muscle nerves, they are always highly specific and can not be accounted for by general target attraction. Specific target attraction, therefore, requires specific cues that cannot be accounted for by the maturation of myotubes alone. In fact, the precursors for limb muscle fibers do not appear to be responsible for muscle identity. While each limb muscle normally develops from a certain subset of somites (Beresford, 1983, Lance-Jones, 1987), somites from foreign axial levels can give rise to normal limb muscles (Chevallier et al., 1977; Chevallier and Kieny, 1982; Beresford, 1983) that are innervated with normal specificity (Lance-Jones, 1987). In addition, in the present study the innervation of limb muscles was specific despite the fact that the precursor population must have regulated substantially following the most extensive dermamyotome deletions. Muscle identity (and, presumably, the corresponding specific target cues) appears to depend on the connective tissue element which is derived from the lateral mesenchyme (Chevallier and Kieny, 1982; Lance-Jones, 1987).

Like limb muscles, the dorsal ramus axons may be able to respond to specific targets. For instance, they may prove to be attracted by epaxial but not limb muscles. The present study did not determine whether subpopulations of dorsal ramus motoneurons are specified for cach epaxial muscle within a segment.

Since the epaxial muscles are serially repeated along much of the anterior-posterior axis, it is possible that the dorsal ramus axons and their targets are segmentally specified. However, even though dorsal ramus axons always grew into small muscle fragments within their own segment, this preference can not be taken as evidence for segmental specificity. A small target, because it is closer, may produce a more effective signal than a target of normal size that is in the adjacent segment. In fact, there is conflicting evidence for segmental specificity. In Xenopus the myotome has been suggested to share a compartmental relationship with the motoneurons that first innervate it (Moody and Jacobson, 1983). In addition, sympathetic preganglionic neurons are able to detect segmental differences in intercostal muscles 
(Wigston and Sanes, 1982, 1985) which are also derived metamerically from the myotomes. However, segmental specificity of innervation in Xenopus was not detected in an in vitro paradigm (McCaig, 1986). If epaxial muscles are segmentally specified by connective tissue, either the sclerotome or the dermatome may be the patterning agent. Both tissues possess regional specificity: the sclerotome will give rise to segment-specific vertebrae even when displaced to other segments (Detwiler, 1934) and the dermis elicits site-specific feather germ patterns even when it is displaced to cranial regions (Noden, 1986). It is not known whether either tissue contributes to the specification of different cpaxial muscles within a segment.

Dorsal ramus axons may be able to respond to cues other than those presented by their target. For instance, these axons appear to detect differences in anterior versus posterior sclerotome. When deprived of target in its own scgment, the dorsal ramus does not move in a straight line directly toward target in an adjacent segment; it extends toward the adjacent segment only when it is dorsal to the bulk of the sclerotome. These growth cones are unlikely to be tracing out the path of diffusion of a molecule from the adjacent target. It is more likely that the growth cones are responding to the anteriorposterior dichotomy in the sclerotome that is responsible for segmentation of outgrowth (see also Tosney, 1987b). When the dorsal ramus has reached a dorsal position it may be above the prohibitory portion of the sclerotome. Alternatively, at this point the target attraction may be strong enough to overcome whatever pathway constraints this environment presents.

Substratum preferences have also been suggested to contribute to the divergence of the dorsal ramus axons from the spinal nerves: dorsal ramus motoneurons might fasciculate less strongly or have a greater preference for sclerotome cells as a substratum than do axons that remain within the spinal nerves (Tosney and Landmesser, 1985a). Were these mechanisms very important to outgrowth, I would have expected to see dorsal ramus growth cones moving into the sclerotome in the absence of the dermamyotome but I did not. In addition, the nonspecific kind of fasciculation that may be mediated by NCAM should be similar in both motoneuron populations, since dorsal ramus and limb motoneurons have comparable levels of NCAM on their surfaces, while both have levels higher than those of DRG (Tosney et al., 1986). The possibility that these axons can display specific neurite interactions of the type known to be important in invertebrates (Raper et al., 1983) has not been examined.

The observations are in accord with the growing conviction that axonal trajectories are a result of the balance of the forces acting on the axons. For instance, axons may abruptly alter their trajectories toward a target when the target-related cue is strong enough to overcome other pathway constraints, such as close neurite-neurite interactions or substratum preferences (see Fraser, 1980; Lance-Jones and Landmesser, 1981a; Tosney and Landmesser, 1985a). It is also probable that the dorsal ramus population normally relies on a simpler set of cues than those axons that must traverse long distances to reach their proper target: a chemotactic cue might suffice for these axons, while cues for sorting out, tracing out permissive pathways, and projecting down correct subsets of these pathways are largely irrelevant to the specificity of their projection.

The sensory component of the dorsal ramus. The results suggest that a dual set of axonal guidance cues guides the outgrowth of the cutaneous component of the dorsal ramus. Chemotaxis of cutaneous afferents to either ectoderm or dermis is insufficient, by itself, to explain outgrowth, since dorsal rami were not seen when ectoderm and dermis were present but epaxial muscle was absent. Conversely, chemotaxis to muscle tissue or fasciculation with motoneurons is insufficient to explain the results, by themselves, since both elements were present when the dorsal rami grew to ventral muscle fragments and yet in these cases cutaneous nerves did not form.

Cutaneous afferents may be initially attracted to dorsal epaxial muscle tissue and subsequently to ectoderm or dermis or they may require a series of substrata for outgrowth, such as motoneuron axons and blood vessels. Any combination of these possibilities is consistent with other evidence. For instance, sensory neurites in culture respond to ectoderm and dermis (Lumsden and Davies, 1983; Verna, 1985; Verna et al., 1986), they can move up a chemotactic gradient (Gunderson and Barrett, 1980) and they can detect adhesive differences in substrata (Letourneau, 1972). In addition, sensory neurites that enter the limb require motoneurons to enter muscle (Landmesser and Honig, 1986; see also Taylor, 1944; Lewis et al., 1981, Swanson, 1985, Scott, 1986). Furthermore, although motoneuron axons in the limb do not slavishly follow blood vessels (Tosney and Landmesser, 1985a), the cutaneous branch of the dorsal ramus (Tosney, 1987a) and the cutaneous nerves in the limb (Landmesser and Honig, 1986) normally develop in close proximity to blood vessels.

Different arrays of cues for different neural populations. The results are in accord with the notion that different populations of neurites that are traversing the same environment respond to some common and some population-specific guidance cues. For instance, both limb and dorsal ramus motor axons appear to prefer anterior over posterior sclerotome for outgrowth. However, additional guidance mechanisms are required for motoneuron axons to reach limb targets, such as distal pathways, specific cues at pathway branches, neuriteneurite interactions, and perhaps general and specific 
target attraction (see Landmesser, 1985, for review). Sensory neurites within the same limb pathways may use very similar cues until they are close to their targets; then they require the axons of motoneurons in order to enter muscle targets and may require blood vessels to form cutaneous branches (Landmesser and Honig, 1986; Honig et al., 1986). Similarly, cutaneous axons in the dorsal ramus do not respond to exactly the same set of cues as motor axons in the same ramus. While dorsal ramus motoneurons require their target, preganglionic axons can navigate in specific directions in the absence of their target (Yip, 1987).

This study provides an interesting test of the hypothesis that a particular motoneuron population can detect specific cues that are invisible to other populations. The only neurites that respond to the dermamyotome are axons in the dorsal ramus and for these axons the dermamyotome is essential for outgrowth. While dorsal ramus axons may ordinarily fasciculate with other axons and preferentially grow out through the anterior sclerotome, these and other navigational aids appear less essential and therefore are less likely to complicate experimental studies of the target-related guidance of this population. The outgrowth of dorsal ramus axons thus provides a system of some promise for the study of specific axonal navigation.

I thank Sophia Tyrrell and Edward Wojcik for technical assistance, Ann Gulley and David Bay for photographic assistance and Richard Hume and Gerhard Schlosser for reading the manuscript. This work was supported by National Institutes of IIealth Grant NS-21308 and a Rackham Faculty Grant from the University of Michigan.

\section{REFERENCES}

BEnNETT, M. R. (1983). Development of neuromuscular synapses. Physiol. Rev. 63, 915-1048.

Bennett, M. R., Davey, D. F., and Uebel, K. E. (1980). The growth of segmental nerves from the brachial myotomes into the proximal muscles of the chick forelimb during development. J. Comp. Neurol. 189, 335-357.

BERESFord, B. (1983). Brachial muscles in the chick embryo: The fate of individual somites J. Embryol. Exp. Morphol. 77, 99-116.

Bentley, D., and Cavidy, M. (1984). Navigational substrates for peripheral pioneer growth cones: Limb-axis polarity cues, limb-segment boundaries and guidepost neurons. Cold Spring Harbor Symp. Quant. Biol., 48, 573-585.

Bovolenta, P., and Mason, C. A. (1985). Growth cones form changes with age and position along the embryonic mouse retinal axon pathway. Soc. Neurosci. Abstr. 11, 176.

CARPEnter, E. M., and Hollyday, M. (1986). Defective innervation of chick limbs in the absence of presumptive Schwann cells. Soc. Neurosci. Abs. 12, 1210 .

Caudy, M., and Bentley, D. (1986). Pioneer growth cone morphologies reveal proximal increases in substrate affinity within leg segments of grasshopper embryos. $J$. Neurosci. 6, 364-379.

Chevallier, A. (1978). Elude de la migration des cellules somitiques dans le mesoderme somatopleural de l'ebauche de l'aile. Wilhelm Ronx's Arch. Dev? Biol. 184, 57-73.
Chevalitier, A. (1979). Role of the somitic mesoderm in the development of the thorax in bird embryos. II. Origin of thoracic and appendicular musculature J. Embryol. Exp. Morphol. 49, 73-88.

Chevaliter, A., and Kieny, M. (1982). On the role of the connective tissue in the patterning of the chick limb musculature. Wilhelm Roux's Arch. Dev. Biol. 191, 277-280.

Chevallier, A., Kieny, M., and Mauger, A. (1977). Limb-somite relationship: Origin of the limb musculature. J. Embryol. Exp. Morphol $41,245-258$.

Chevallier, A., Kieny, M., and Mavger, A. (1978). Limb-somite relationship: Effect of removal of somitic mesoderm on the wing musculature. J. Embryol. Exp. Morphol. 43, 263-278.

CHRIST, B., JACOB, M., and JACOB, H. J. (1983). On the origin and development of the ventrolateral abdominal muscles in the avian embryo. An experimental and ultrastructural study. Anat. Embryol. 166, 87-101.

CRICK, F. (1970). Diffusion in embryogenesis. Nature (London) 31, 420422.

DeTwILER, S. R. (1934). An experimental study of spinal nerve segmentation in Ablystoma with reference to the plurisegmental contribution to the brachial plexus. J. Exp. Zool. 67, 395-441.

Ferguson, B. (1983). Development of motor innervation of the chick following dorsal-ventral limb bud rotations. J. Neurosci. 3, 17601772 .

FRASER, S. E. (1980). A differential adhesion approach to the patterning of nerve connections. Dev. Biol. 79, 453-464.

GundERSON, R. W., and BARRETT, J. N. (1980). Characterization of the turning response with dorsal root neurites toward nerve growth factor. J. Cell Biol. 87, 546-554.

HambUrger, V., and HamiLton, H. L. (1951). A series of normal stages in the development of the chick embryo. J. Morphol. 88, 49-82.

HOLLYDAY, M. (1983). Development of motor innervation of chick limbs In "Limb Development and Regeneration" Part A, pp. 183-193. A. R. Liss, NY.

HoNIG, M. G. (1982). The development of sensory projection pattern in the embryonic chick hindlimb. J. Physiol. (Lomdom) 330, 175-202.

Honig, M. G., LANCE-Jones, C., and LANDMEsser, L. (1986). The development of sensory projection patterns in embryonic chick hindlimb under experimental conditions. Dev. Biol. 118, 532-548.

JACOB, M., Christ, B., and JACOB, H. J. (1979). The migration of myogenic cells from the somites into the leg region of avian embryos. An ultrastructural study. Anat. Embryol. 157, 291-309.

KEYNES, R. J., and Stern, C. D. (1984). Segmentation in the vertebrate nervous system. Nature (Lrmdom) 310, 786-789.

LANCE-JONES, C. J. (1986). Motoneuron projection patterns in chick embryonic limbs with a double complement of dorsal thigh musculature. Dev. Biol. 116, 387-406.

LANCE-JONES, C. J. (1987). Motoneuron axon guidance: The development of specific projections to two muscles in the embryonic chick limb. Brain Behav. Evolution, in press.

LANCE-Jones, C. J., and LANDMESSER, L. T. (1980). Motoneuron projection patterns in the chick hindlimb following early partial spinal cord reversals. J. Physiol. 302, 581-602.

LANCE-Jones, C. J., and LANDmesser, L. T. (1981a). Pathway selection by embryonic chick motoneurons in an experimentally altered environment. Proc. $R$. Soc. Lomdm R. 214, 19-52.

LANCE-JoNeS, C. J., and LANDMESSER, L. T. (1981b). Pathway selection by chick lumbosacral motoneurons during normal development. Proc R. Soc. London B. 214, 1-18.

Landmesser, L. T. (1978). The development of motor projection patterns in the chick hindlimb. J. Physiol. 284, 391-414.

LANDMESsER, L. T. (1985). The development of specific motor pathways in the chick embryo. Trends Neurosci. 7, 336339.

LANDMESSER, L. T. (1986). Axonal guidance and the formation of neural circuits. Trends Neurosei. 9, 489-492. 
LANDMEsSER, L. T. (1987). Peripheral guidance cues and the formation of specific motor projections in the chick. In "From Message to Mind: Directions in Developmental Neurobiology." In press.

LANDMESSER, L., and HoNIG, M. G. (1986). Altered sensory projections in the chick hind limb following the early removal of motoneurons. Dev. Biol. 118, 511-531.

LeTOURneau, P. C. (1972). Cell-to-substratum adhesion and guidance of axonal elongation. Dev. Biol. 44, 92-101.

Lewis, J., Al-Ghaith, L., Swanson, G., and Khan, A. (1983). The control of axon outgrowth in the developing chick wing. In "Limb Development and Regeneration" Part A, pp. 195-205. A. R. Liss, NY.

Lewis, J., Chevallier, A., Kieny, M., and WolPert, L. (1981). Muscle nerve branches do not develop in chick wings devoid of muscle. $J$. Embryol. Exp. Morphol. 64, 211-232.

LoRing, J. F., and ERICKson, C. A. (1986). Patterns of neural crest migration in the chick embryo. Soc. Neurosci. Abstr. 12, 1209.

LoRING, J. F., and Erickson, C. A. (1987). Neural crest cell migratory pathways in the chick embryo. Dev. Biol. 121, 220-236.

Lumsden, A. G. S., and DAviEs, A. M. (1983). Earliest sensory nerve fibers are guided to peripheral targets by attractants other than nerve growth factor. Nature (London) 306, 786-788.

MAUGER, A. (1972). Role du mesoderme somitique dans le developpement du plumage dorsal chez l'embryon de Poulet. I. Origine, capacities de regulation et determination du mesoderme plumigene. J. Embryol. Exp. Morphol. 28, 313-341.

MAUGER, A. M., and KIENY, M. (1980). Migration and organogenetic capacities of muscle cells in bird embryos. Wilhelm Roux's Arch. Dev. Biol. 189, 123-134

MCCAIG, C. D. (1986). Myoblasts and myoblast-conditioned medium attract the earliest spinal neurites from frog embryos. J. Physiol. 375, 39-54.

MoODY, S., and JACOBSON, S. (1983). Compartmental relationship between anuran primary spinal motoneurons and somitic muscle fibers that they first innervate. $J$. Neurosci. 3, 1670-1682.

NeWgreEN, D. F., SCHÉL, M., and KASTNER, V. (1986). Morphogenesis of sclerotome and neural crest in avian embryos. In vivo and in vitro studies on the role of notochordal extracellular material. Cell Tissue Res. 244, 299-313.

NoAkes, P. G., EveretT, A. W., and BenNeTt, M. R. (1986). The growth of muscle nerves in relation to the formation of primary myotubes in the developing chick forelimb. J. Comp. Neurol. 248, 245-256.

NoDEn, D. M. (1986). Patterning of avian craniofacial muscles. Dev. Biol. 116, 347-356.

Phelan, K., and Holdyday, M. (1986). Pathway selection in muscleless chick limbs. Soc. Neurosci. Abstr. 12, 1210.

Raper, J. A., Bastiani, M., and Goodman, C. S. (1983). Pathfinding by neuronal growth cones in grasshopper embryos. II. Selective fasciculation onto specific axonal pathways. $J$. Neurosci. 3, 31-41.

RickmanN, M., FAUCET, J. W., and KeYNES, R. J. (1985). The migration of neural crest cells and the growth of motor axons through the rostral half of the chick somite. J. Embryol. Exp. Morphol. 90, 437455.

ROBERTS, A., and TAYLOR, J. S. H. (1983). A study of the growth cones of developing embryonic sensory neurites. I. Embryol Exp. Morphol. $75,31-47$.

ScotT, S. A. (1984). The effects of neural crest deletions on the development of sensory innervation patterns in embryonic chick hind limb. J. Physiol. 352, 385-404.

ScoTT, S. A. (1986). Skin sensory innervation patterns in embryonic chick hindlimb following dorsal root ganglion reversal. J. Neurobiol. $17,649-668$.

Stern, C. D., Sisodiya, S. M., and Keynes, R. J. (1986). Interactions between neurites and somite cells: Inhibition and stimulation of nerve growth in the chick embryo. J. Embryol. Exp. Morphol. 91, $209-226$.
Swanson, G. J. (1985). Paths taken by sensory nerve fibres in aneural chick wing buds. J. Embryol. Exp. Morphol. 86, 109-124.

Summerbell, D., and Stirling, R. V. (1981). The innervation of dorsoventrally reversed chick wings: Evidence that motor axons do not actively seek out their appropriate targets. J. Embryol. Exp. Morphol. 61, 233-247.

Taghert, P. H., Bastiani, M. J., Ho, R. K., and Goodman, C. S. (1982), Guidance of pioneer growth cones: Filopodial contacts and coupling revealed with an antibody to lucifer yellow. Dev. Biol. 94, 391-399.

TANAKA, H., and LANDMEsser, L. (1987). Potential axonal guidance features in the chick limb revealed by antibodies to neural crest and extracellular matrix molecules. Dev. Biol., in press.

TAYLOR, A. C. (1944). Selectivity of nerve fibers from the dorsal and ventral roots in the development of the frog limb. J. Exp. Zool. 96, 159-185.

Tosney, K. W. (1986). Proximal tissues and patterned neurite outgrowth into the chick hindlimb. Soc. Neurosci. Abstr. 12, 1210.

Tosney, K. W. (1987a). Proximal tissues and patterned neurite outgrowth at the lumbosacral level of the chick embryo: Development of the dorsal ramus and the epaxial muscles. In preparation.

Tosney, K. W. (1987b). Proximal tissues and patterned neurite outgrowth at the lumbosacral level of the chick embryo: Deletion of the somite. In preparation.

Tosney, K. W., and LANDMEsSer, L. T. (1984). Pattern and specificity of axonal outgrowth following varying degrees of chick limb bud ablation. J. Neurosci. 4, 2158-2527.

TOSney, K. W., and Landmesser, L. T. (1985a). Development of the major pathways for neurite outgrowth in the chick hindlimb. Dev. Biol. 109, 193-214.

Tosney, K. W., and Landmesser, L. T. (1985b). Specificity of motoneuron growth cone outgrowth in the chick embryo. J. Neurosci. 5, 2336-2344.

TosNeY, K. W., and LANDMESSER, L. T. (1985c). Growth cone morphology and trajectory in the lumbosacral region of the chick embryo. $J$. Neurosei. 5, 2345-2358.

Tosney, K. W., and Landmesser, L. T. (1986). Neurites and growth cones in the chick embryo. Enhanced tissue preservation and visualization of HRP-labeled subpopulations in serial $25 \mu \mathrm{m}$ plastic sections cut on a rotary microtome. J. Histochem. Cytochem. 34, 953957.

Tosney, K. W., Watanabe, M., Landmesser, L., and Rutishauser, U. (1986). The distribution of NCAM in the chick hindlimb during axon outgrowth and synaptogenesis. Dev. Biol. 114, 437-452.

VERNA, J.-M. (1985). In vitro analysis of interactions between sensory neurons and skin: Evidence for selective innervation of dermis and epidermis. J. Embryol. Exp. Morphol. 86, 53-70.

VERNA, J.-M., UsSON, Y., and SAXOD, R. (1986). Differential growth of sensory neurons in vitro in presence of dermis and epidermis. A quantitative time-lapse analysis. Cell Differ. 18, 183-188.

WHITELAW, V., and HOLLYDAY, M. (1983a). Neural pathway constraints in the motor innervation of the chick hindlimb following dorsoventral rotations of distal limb segments. J. Neurosci. 3, 1226-1233.

Whitelaw, V., and Hollyday, M. (1983b). Position-dependent motor innervation of the chick hindlimb following serial and parallel duplications of limb segments. J. Neurosei. 3, 1216-1225.

Wigston, D., and SANES, J. (1982). Selective reinnervation of adult mammalian muscles by axons from different segmental levels. $\mathrm{Na}$ ture (London) 299, 464-467.

Wigston, D. J., and SANES, J. R. (1985). Selective reinnervation of intercostal muscles transplanted from different segmental levels to a common site. J. Neurosci. 5, 1208-1221.

YIP, J. (1986). Axonal guidance in avian sympathetic preganglionic neurons. Soc. Neurosci. Abstr. 12, 1503.

YIP, J. (1987). Target cues are not required for the guidance of sympathetic preganglionic axons. Dev. Brain Res. 32, 155-159. 